\title{
The Lattice of Subvarieties of Semilattice Ordered Algebras
}

\author{
A. Pilitowska - A. Zamojska-Dzienio
}

Received: 27 September 2012 / Accepted: 30 May 2013 / Published online: 20 June 2013

(C) The Author(s) 2013. This article is published with open access at Springerlink.com

\begin{abstract}
This paper is devoted to the semilattice ordered $\mathcal{V}$-algebras of the form $(A, \Omega,+)$, where + is a join-semilattice operation and $(A, \Omega)$ is an algebra from some given variety $\mathcal{V}$. We characterize the free semilattice ordered algebras using the concept of extended power algebras. Next we apply the result to describe the lattice of subvarieties of the variety of semilattice ordered $\mathcal{V}$-algebras in relation to the lattice of subvarieties of the variety $\mathcal{V}$.
\end{abstract}

Keywords Ordered structures • Semilattices • Power algebras • Free algebras • Fully invariant congruences $\cdot$ Varieties $\cdot$ Lattice of subvarieties

Mathematics Subject Classifications (2010) 08B15 • 08B20 • 08A30 • 06A12 • 06F25

\section{Introduction}

Ordered algebraic structures, particularly ordered fields, ordered vector spaces, ordered groups and semigroups, have a well-established tradition in mathematics. It is due to their internal interest and due to their applications in other areas.

Special class of ordered algebras is given by semilattice ordered ones.

Let $\mho$ be the variety of all algebras $(A, \Omega)$ of finitary type $\tau: \Omega \rightarrow \mathbb{N}$ and let $\mathcal{V} \subseteq \mho$ be a subvariety of $\mho$.

While working on this paper, the authors were supported by the Statutory Grant of Warsaw University of Technology 504P/1120/0025/000.

A. Pilitowska · A. Zamojska-Dzienio $(\varangle)$

Faculty of Mathematics and Information Science,

Warsaw University of Technology, ul. Koszykowa 75, 00-662 Warsaw, Poland e-mail: azamojsk@elka.pw.edu.pl

URL: http://www.mini.pw.edu.pl/ azamojsk

A. Pilitowska

e-mail: apili@mini.pw.edu.pl

URL: http://www.mini.pw.edu.pl/ apili 
Definition 1.1 An algebra $(A, \Omega,+)$ is called a semilattice ordered $\mathcal{V}$-algebra (or briefly semilattice ordered algebra) if $(A, \Omega)$ belongs to a variety $\mathcal{V},(A,+)$ is a (join) semilattice (with semilattice order $\leq$, i.e. $x \leq y \Leftrightarrow x+y=y$ ), and the operations from the set $\Omega$ distribute over the operation + , i.e. for each $0 \neq n$-ary operation $\omega \in \Omega$, and $x_{1}, \ldots, x_{i}, y_{i}, \ldots, x_{n} \in A$

$$
\begin{aligned}
\omega\left(x_{1}, \ldots, x_{i}+y_{i}, \ldots, x_{n}\right)= & \omega\left(x_{1}, \ldots, x_{i}, \ldots, x_{n}\right) \\
& +\omega\left(x_{1}, \ldots, y_{i}, \ldots, x_{n}\right),
\end{aligned}
$$

for any $1 \leq i \leq n$.

Basic examples of semilattice ordered algebras are provided by additively idempotent semirings, distributive lattices, semilattice ordered semigroups and modals (semilattice ordered idempotent and entropic algebras).

In 1979 McKenzie and Romanowska [10] showed that there are exactly five varieties of dissemilattices-semilattice ordered semilattices. Apart from the variety of all dissemilattices and the trivial variety, there are the variety of distributive lattices, the variety of "stammered" semilattices (where both basic semilattice operations are equal) and the variety of distributive dissemilattices.

In 2005 Ghosh et al. [5] described the lattice of all subvarieties of the variety generated by all ordered bands (semirings whose multiplicative reduct is an idempotent semigroup and additive reduct is a chain). They showed that the lattice is distributive and contains precisely 78 varieties. Each of them is finitely based and generated by a finite number of finite ordered bands.

In the same year, Kuřil and Polák [9] introduced the certain closure operators on relatively free semigroup reducts and applied them to describe the lattice of subvarieties of the variety of all semilattice ordered semigroups.

But, in general, very little is known about varieties of modals. In 1995 Kearnes [8] proved that to each variety $\mathcal{V}$ of entropic modals one can associate a commutative semiring $\mathbf{R}(\mathcal{V})$, whose structure determines many of the properties of the variety. In particular,

Theorem 1.2 ([8]) The lattice of subvarieties of the variety $\mathcal{V}$ of entropic modals is dually isomorphic to the congruence lattice $\operatorname{Con} \mathbf{R}(\mathcal{V})$ of the semiring $\mathbf{R}(\mathcal{V})$.

Using Theorem 1.2, Ślusarska [17] described the lattice of subvarieties of entropic modals $(D, \cdot,+)$ whose groupoid reducts $(D, \cdot)$ satisfy the additional identity: $x(y z) \approx x y$.

Just recently (see [13]) we described some family of fully invariant congruences on the free semilattice ordered idempotent and entropic algebras which can partially result in describing subvariety lattices of modals.

The main aim of this paper is to describe a structure of the subvariety lattice of all semilattice ordered $\mho$-algebras of a given type and study how it is related to the subvariety lattice of all $\mho$-algebras. Kuřil and Polák introduced in [9] the notion of an admissible closure operator and applied it to describe the subvariety lattice of the variety of semilattice ordered semigroups. Description proposed by them is complicated and strictly depends on properties of semigroups. Applying the techniques from power algebras theory we simplify their description. Moreover, 
we generalize their results and obtain another type of description of the subvariety lattice of all semilattice ordered algebras.

We start with the following easy observation.

Lemma 1.3 Let $(A, \Omega,+)$ be a semilattice ordered $\mho$-algebra, $x_{1}, \ldots, x_{n}, y_{1}, \ldots, y_{n} \in$ $A$, and let $\omega \in \Omega$ be an $0 \neq n$-ary operation. If $x_{i} \leq y_{i}$ for each $1 \leq i \leq n$, then

$$
\omega\left(x_{1}, \ldots, x_{n}\right) \leq \omega\left(y_{1}, \ldots, y_{n}\right) .
$$

Note, that by Lemma 1.3 each semilattice ordered $\mho$-algebra $(A, \Omega,+)$ is an ordered algebra $(A, \Omega, \leq)$ in the sense of $[1,3,4]$.

There are two other basic properties worth mentioning.

Lemma 1.4 Let $(A, \Omega,+)$ be a semilattice ordered $\mho$-algebra, and $x_{i j} \in A$ for $1 \leq i \leq$ $n, 1 \leq j \leq r$. Then for each $0 \neq n$-ary operation $\omega \in \Omega$ we have

$$
\begin{aligned}
& \omega\left(x_{11}, \ldots, x_{n 1}\right)+\ldots+\omega\left(x_{1 r}, \ldots, x_{n r}\right) \\
\leq & \omega\left(x_{11}+\ldots+x_{1 r}, \ldots, x_{n 1}+\ldots+x_{n r}\right) .
\end{aligned}
$$

Lemma 1.5 Let $(A, \Omega,+)$ be a semilattice ordered $\mho$-algebra and let $\omega \in \Omega$ be an $0 \neq$ $n$-ary operation. The algebra $(A, \Omega,+)$ satisfies for any $x \in A$ the condition

$$
\omega(x, \ldots, x) \leq x,
$$

if and only if for any $x_{1}, \ldots, x_{n} \in A$ the following holds

$$
\omega\left(x_{1}, \ldots, x_{n}\right) \leq x_{1}+\ldots+x_{n} .
$$

In particular, if a semilattice ordered $\mho$-algebra $(A, \Omega,+)$ is idempotent then Eq. 1.5 holds.

It is easy to see that in general both Eqs. 1.2 and 1.3 hold also for term operations. The proofs go just by induction on the complexity of terms. Also, in such a case, for any term operation $t$, we obtain the inequality

$$
t\left(x_{1}, \ldots, x_{i}, \ldots, x_{n}\right)+t\left(x_{1}, \ldots, y_{i}, \ldots, x_{n}\right) \leq t\left(x_{1}, \ldots, x_{i}+y_{i}, \ldots, x_{n}\right),
$$

that generalizes the distributive law Eq. 1.1.

The paper is organized as follows. In Section 2 examples of semilattice ordered $\mathcal{V}$-algebras are provided, for some given varieties $\mathcal{V}$. Among the others, we mention semilattice ordered $n$-semigroups, extended power algebras and modals. In Section 3 we describe the free semilattice ordered algebras (Theorem 3.4) and next we apply the result to describe the identities which are satisfied in varieties of semilattice ordered algebras (Corollary 3.10). Section 4 is devoted to present the "main knots" in the subvariety lattice of semilattice ordered algebras (Theorem 4.7). In Section 5 we characterize so called $\mathcal{V}$-preserved subvarieties of the variety of all semilattice ordered algebras. In Theorem 5.9 we show that there is a correspondence between the set of all $\mathcal{V}$-preserved subvarieties and the set of some fully invariant congruence relations on the extended power algebra of the $\mathcal{V}$-free algebra. Theorem 5.12 contains the main result of this paper, i.e. the full description of the lattice of all subvarieties of the variety of all semilattice ordered algebras. The last Section 6 summarizes all results in the convenient form of the algorithm. 
The notation $t\left(x_{1}, \ldots, x_{n}\right)$ means the term $t$ of the language of a variety $\mathcal{V}$ contains no other variables than $x_{1}, \ldots, x_{n}$ (but not necessarily all of them). All operations considered in the paper are supposed to be finitary. We are interested here only in varieties of algebras, so the notation $\mathcal{W} \subseteq \mathcal{V}$ means that $\mathcal{W}$ is a subvariety of a variety $\mathcal{V}$.

\section{Examples}

In this section we discuss some basic and natural examples of semilattice ordered algebras.

Example 2.1 Semilattice ordered semigroups. An algebra $(A, \cdot,+)$, where $(A, \cdot)$ is a semigroup, $(A,+)$ is a semilattice and for any $a, b, c \in A, a \cdot(b+c)=a \cdot b+$ $a \cdot c$ and $(a+b) \cdot c=a \cdot c+b \cdot c$ is a semilattice ordered semigroup. In particular, semirings with an idempotent additive reduct [11,18], distributive bisemilattices [10] and distributive lattices are semilattice ordered $\mathcal{S G}$-algebras, where $\mathcal{S G}$ denotes the variety of all semigroups.

Example 2.2 Semilattice ordered $\boldsymbol{n}$-semigroups. Let $2 \leq n \in \mathbb{N}$. An algebra $(A, f)$ with one $n$-ary operation $f$ is called an $n$-semigroup, if the following associative laws hold:

$$
\begin{aligned}
& f\left(f\left(a_{1}, \ldots, a_{n}\right), a_{n+1}, \ldots, a_{2 n-1}\right) \\
&=\cdots=f\left(a_{1}, \ldots, a_{i}, f\left(a_{i+1}, \ldots, a_{i+n}\right), a_{i+n+1}, \ldots, a_{2 n-1}\right) \\
& \quad=\ldots=f\left(a_{1}, \ldots, a_{n-1}, f\left(a_{n}, \ldots, a_{2 n-1}\right)\right),
\end{aligned}
$$

for all $a_{1}, \ldots, a_{2 n-1} \in A$. A semigroup is a 2 -semigroup in this sense.

An algebra $(A, f,+)$, where $(A, f)$ is an $n$-semigroup, $(A,+)$ is a semilattice and the Eq. 1.1 are satisfied for the operation $f$, is a semilattice ordered $n$-semigroup.

Let $\mathcal{S G}_{n}$ be the variety of all $n$-semigroups, $(A,+)$ be a semilattice and $\operatorname{End}(A,+)$ be the set of all endomorphisms of $(A,+)$. For each $2 \leq n \in \mathbb{N}$, we define $n$-ary composition

$$
\omega_{n}:(\operatorname{End}(A,+))^{n} \rightarrow \operatorname{End}(A,+), \omega_{n}\left(f_{1}, \ldots, f_{n}\right):=f_{n} \circ \ldots \circ f_{1} .
$$

Then the algebra $\left(\operatorname{End}(A,+), \omega_{n}, \vee\right)$, with $\left(f_{1} \vee f_{2}\right)(x):=f_{1}(x)+f_{2}(x)$, for $x \in$ $A$, is a semilattice ordered $n$-semigroup (semilattice ordered $\mathcal{S G}_{n}$-algebra). The algebra $\left(\operatorname{End}(A,+),\left\{\omega_{n}: n \in \mathbb{N}\right\}, \vee\right)$ is also an example of a semilattice ordered algebra.

Another example of a semilattice ordered $n$-semigroup can be obtained as follows. Let $A$ be a set and $\operatorname{Rel}(A)$ be the set of all binary relations on $A$. For each $2 \leq n \in \mathbb{N}$, we define $n$-ary relational product

$$
\omega_{n}:(\operatorname{Rel}(A))^{n} \rightarrow \operatorname{Rel}(A), \quad \omega_{n}\left(r_{1}, \ldots, r_{n}\right):=r_{n} \circ \ldots \circ r_{1} .
$$

Then the algebra $\left(\operatorname{Rel}(A), \omega_{n}, \cup\right)$, where $\cup$ is a set union, is a semilattice ordered $\mathcal{S G}_{n}$-algebra and the algebra $\left(\operatorname{Rel}(A),\left\{\omega_{n}: n \in \mathbb{N}\right\}, \cup\right)$ is a semilattice ordered algebra. 
Example 2.3 Extended power algebras. For a given set $A$ denote by $\mathcal{P}_{>0} A$ the family of all non-empty subsets of $A$. For any $n$-ary operation $\omega: A^{n} \rightarrow A$ we define the complex operation $\omega:\left(\mathcal{P}_{>0} A\right)^{n} \rightarrow \mathcal{P}_{>0} A$ in the following way:

$$
\omega\left(A_{1}, \ldots, A_{n}\right):=\left\{\omega\left(a_{1}, \ldots, a_{n}\right) \mid a_{i} \in A_{i}\right\},
$$

where $\emptyset \neq A_{1}, \ldots, A_{n} \subseteq A$. The set $\mathcal{P}_{>0} A$ also carries a join semilattice structure under the set-theoretical union $\cup$. Jónsson and Tarski proved in [7] that complex operations distribute over the union $\cup$. Hence, for any algebra $(A, \Omega) \in \mho$, the extended power algebra $\left(\mathcal{P}_{>0} A, \Omega, \cup\right)$ is a semilattice ordered $\mho$-algebra. The algebra $\left(\mathcal{P}_{>0}^{<\omega} A, \Omega, \cup\right)$ of all finite non-empty subsets of $A$ is a subalgebra of $\left(\mathcal{P}_{>0} A, \Omega, \cup\right)$.

Example 2.4 Modals. An idempotent (in the sense that each singleton is a subalgebra) and entropic algebra (any two of its operations commute) is called a mode. Let $\mathcal{M}$ denote the variety of all modes. A modal is an algebra $(M, \Omega,+)$, such that $(M, \Omega) \in \mathcal{M},(M,+)$ is a (join) semilattice, and the $\Omega$ operations distribute over + . Examples of modals include distributive lattices, dissemilattices [10]—algebras $(M, \cdot,+)$ with two semilattice structures $(M, \cdot)$ and $(M,+)$ in which the operation . distributes over the operation + , and the algebra $\left(\mathbb{R}, \underline{I}^{0}\right.$, max $)$ defined on the set of real numbers, where $\underline{I}^{0}$ is the set of the following binary operations: $p: \mathbb{R} \times \mathbb{R} \rightarrow \mathbb{R}$; $(x, y) \mapsto(1-p) x+p y$, for each $p \in(0,1) \subset \mathbb{R}$.

Each modal is in fact a semilattice ordered $\mathcal{M}$-algebra. Modes and modals were introduced and investigated in detail by Romanowska and Smith $[15,16]$.

If a modal $(M, \Omega,+)$ is entropic, then $(M, \Omega,+)$ is a mode and it is an example of a semilattice mode. Semilattice modes were described by Kearnes in [8].

\section{Free Semilattice Ordered Algebras and Identities}

Results of Kurril and Polák [9] show that the problem of the characterization of semilattice ordered algebras requires the knowledge of the structure of the power algebra of a given algebra. In this section we will describe the free semilattice ordered algebras using the concept of extended power algebras. Next we will apply the result to describe the identities which are satisfied in varieties of semilattice ordered algebras.

Let $\mho$ be the variety of all algebras of finitary type $\tau: \Omega \rightarrow \mathbb{N}$ and let $\mathcal{V}$ be a subvariety of $\mho$. Let $\left(F_{\mathcal{V}}(X), \Omega\right)$ be the free algebra over a set $X$ in the variety $\mathcal{V}$ and let $\mathcal{S}_{\mathcal{V}}$ denote the variety of all semilattice ordered $\mathcal{V}$-algebras.

Theorem 3.1 (Universality Property for Semilattice Ordered Algebras) Let $X$ be an arbitrary set and $(A, \Omega,+) \in \mathcal{S}_{\mathcal{V}}$. Each mapping $h: X \rightarrow A$ can be extended to a unique homomorphism $\overline{\bar{h}}:\left(\mathcal{P}_{>0}^{<\omega} F_{\mathcal{V}}(X), \Omega, \cup\right) \rightarrow(A, \Omega,+)$, such that $\overline{\bar{h}} /_{X}=h$.

Proof Let $(A, \Omega,+) \in \mathcal{S}_{\mathcal{V}}$. By assumption, $(A, \Omega) \in \mathcal{V}$. So any mapping $h: X \rightarrow A$ may be uniquely extended to an $\Omega$-homomorphism $\bar{h}:\left(F_{\mathcal{V}}(X), \Omega\right) \rightarrow(A, \Omega)$.

Further, $\Omega$-homomorphism $\bar{h}$ can be extended to a unique $\{\Omega, \cup\}$-homomorphism

$$
\overline{\bar{h}}:\left(\mathcal{P}_{>0}^{<\omega} F_{\mathcal{V}}(X), \Omega, \cup\right) \rightarrow(A, \Omega,+) ; \quad \overline{\bar{h}}(T) \mapsto \sum_{t \in T} \bar{h}(t),
$$


where $T$ is a non-empty finite subset of $F_{\mathcal{V}}(X)$.

To show that $\overline{\bar{h}}$ is an $\Omega$-homomorphism, consider an $n$-ary operation $\omega \in \Omega$ and non-empty finite subsets $T_{1}, \ldots, T_{n} \subseteq F_{\mathcal{V}}(X)$. Then

$$
\begin{aligned}
& \overline{\bar{h}}\left(\omega\left(T_{1}, \ldots, T_{n}\right)\right)=\overline{\bar{h}}\left(\left\{\omega\left(t_{1}, \ldots, t_{n}\right) \mid t_{i} \in T_{i}\right\}\right) \\
& =\sum_{\left(t_{1}, \ldots, t_{n}\right) \in T_{1} \times \ldots \times T_{n}} \bar{h}\left(\omega\left(t_{1}, \ldots, t_{n}\right)\right) \\
& =\sum_{\left(t_{1}, \ldots, t_{n}\right) \in T_{1} \times \ldots \times T_{n}} \omega\left(\bar{h}\left(t_{1}\right), \ldots, \bar{h}\left(t_{n}\right)\right) \\
& =\omega\left(\sum_{t_{1} \in T_{1}} \bar{h}\left(t_{1}\right), \ldots, \sum_{t_{n} \in T_{n}} \bar{h}\left(t_{n}\right)\right)=\omega\left(\overline{\bar{h}}\left(T_{1}\right), \ldots, \overline{\bar{h}}\left(T_{n}\right)\right) .
\end{aligned}
$$

Moreover, $\overline{\bar{h}}$ is a semilattice homomorphism because

$$
\overline{\bar{h}}\left(T_{1} \cup T_{2}\right)=\sum_{t \in T_{1} \cup T_{2}} \bar{h}(t)=\sum_{t_{1} \in T_{1}} \bar{h}\left(t_{1}\right)+\sum_{t_{2} \in T_{2}} \bar{h}\left(t_{2}\right)=\overline{\bar{h}}\left(T_{1}\right)+\overline{\bar{h}}\left(T_{2}\right) .
$$

The uniqueness of $\overline{\bar{h}}$ is obvious.

By Theorem 3.1, for an arbitrary variety $\mathcal{V} \subseteq \mathcal{V}$, the algebra $\left(\mathcal{P}_{>0}^{<\omega} F_{\mathcal{V}}(X), \Omega, \cup\right)$ has the universality property for semilattice ordered algebras in $\mathcal{S}_{\mathcal{V}}$, but in general, the algebra itself doesn't have to belong to the variety $\mathcal{S}_{\mathcal{V}}$.

Example 3.2 Let $\mathcal{V}$ be a variety of idempotent groupoids satisfying the identities:

$$
x \cdot(y \cdot z) \approx x \cdot y \approx(x \cdot y) \cdot x \text { and }(x \cdot y) \cdot y \approx x .
$$

Consider the free groupoid $\left(F_{\mathcal{V}}(X), \cdot\right)$ over a set $X$ in the variety $\mathcal{V}$ and its two generators $x, y \in X$. One can easily see that

$$
(\{x\}\{x, y, x y, y x\})\{x, y, x y, y x\}=\{x, x y\} \neq\{x\} .
$$

This shows that the algebra $\left(\mathcal{P}_{>0}^{<\omega} F_{\mathcal{V}}(X), \cdot, \cup\right)$ does not belong to the variety $\mathcal{S}_{\mathcal{V}}$.

Note that the semilattice ordered algebra $\left(\mathcal{P}_{>0}^{<\omega} F_{\mathcal{V}}(X), \Omega, \cup\right)$ is generated by the set $\{\{x\} \mid x \in X\}$. Hence, if $\left(\mathcal{P}_{>0}^{<\omega} F_{\mathcal{V}}(X), \Omega, \cup\right) \in \mathcal{S}_{\mathcal{V}}$, then it is, up to isomorphism, the unique algebra in $\mathcal{S}_{\mathcal{V}}$ generated by a set $X$, with the universal mapping property.

Corollary 3.3 The semilattice ordered algebra $\left(\mathcal{P}_{>0}^{<\omega} F_{\mathcal{V}}(X), \Omega, \cup\right)$ is free over a set $X$ in the variety $\mathcal{S}_{\mathcal{V}}$ if and only if $\left(\mathcal{P}_{>0}^{<\omega} F_{\mathcal{V}}(X), \Omega, \cup\right) \in \mathcal{S}_{\mathcal{V}}$.

In particular, one obtains

Theorem 3.4 The semilattice ordered algebra $\left(\mathcal{P}_{>0}^{<\omega} F_{\mho}(X), \Omega, \cup\right)$ is free over a set $X$ in the variety $\mathcal{S}_{\mho}$. Moreover, $\mathcal{S}_{\mho}=\operatorname{HSP}\left(\left(\mathcal{P}_{>0}^{<\omega} \mathrm{F}_{\mho}(\mathrm{X}), \Omega, \cup\right)\right)$ for an infinite set $X$. 
With each subvariety $\mathcal{S} \subseteq \mathcal{S}_{\mho}$ of semilattice ordered $\mho$-algebras we can associate a least subvariety $\mathcal{V}$ of $\mho \mathcal{V}$ with the property $\mathcal{S} \subseteq \mathcal{S}_{\mathcal{V}}$. But, for two different subvarieties $\mathcal{V}$ and $\mathcal{W}$ of $\mathcal{V}$, the varieties $\mathcal{S}_{\mathcal{V}}$ and $\mathcal{S}_{\mathcal{W}}$ can be equal.

Example 3.5 A differential groupoid is a mode groupoid $(D, \cdot)$ satisfying the additional identity:

$$
x(y z) \approx x y .
$$

Each proper non-trivial subvariety of the variety $\mathcal{D}$ of differential groupoids (see [14]) is relatively based by a unique identity of the form

$$
\left(\ldots \left((x \underbrace{y) y) \ldots) y}_{i-\text { times }}=: x y^{i} \approx x y^{i+j}\right.\right.
$$

for some $i \in \mathbb{N}$ and positive integer $j$. Denote such a variety by $\mathcal{D}_{i, i+j}$. Obviously, the variety $\mathcal{D}_{0,1}$ is exactly the variety $\mathcal{L} \mathcal{Z}$ of left-zero semigroups (groupoids $(A, \cdot)$ such that $a \cdot b=a$ for all $a, b \in A$ ).

Let $\mathcal{S}_{\mathcal{D}}$ denote the variety of all differential modals (modals whose mode reduct is a differential groupoid) and let $\mathcal{S}_{\mathcal{D}_{0, j}} \subseteq \mathcal{S}_{\mathcal{D}}$ be the variety of semilattice ordered $\mathcal{D}_{0, j^{-}}$ groupoids. We showed in [12] that for each positive integer $j$, one has $\mathcal{S}_{\mathcal{D}_{0, j}}=\mathcal{S}_{\mathcal{L Z}}$.

Example above arises the question, for which different subvarieties $\mathcal{V}_{1} \neq \mathcal{V}_{2} \subseteq \mho$, the varieties $\mathcal{S}_{\mathcal{V}_{1}}$ and $\mathcal{S}_{\mathcal{V}_{2}}$ are different, too.

To answer this, for an arbitrary binary relation $\Theta$ on the set $\mathcal{P}_{>0}^{<\omega} F_{\mathcal{V}}(X)$ we will introduce a new binary relation $\widetilde{\Theta} \subseteq F_{\mathcal{V}}(X) \times F_{\mathcal{V}}(X)$. For $t, u \in F_{\mathcal{V}}(X)$

$$
(t, u) \in \widetilde{\Theta} \Leftrightarrow(\{t\},\{u\}) \in \Theta .
$$

Example 3.6 It is easy to see that for the least equivalence relation $i d_{\mathcal{P}}^{<\omega 0} F_{\mathcal{V}}(X)$ and the greatest equivalence relation $\mathcal{T}$ on the set $\mathcal{P}_{>0}^{<\omega} F_{\mathcal{V}}(X)$, we have $\widetilde{i d}_{\mathcal{P}>0}^{<\omega} F_{\mathcal{V}}(X)=i d_{F_{\mathcal{V}}(X)}$ and $\widetilde{\mathcal{T}}=F_{\mathcal{V}}(X) \times F_{\mathcal{V}}(X)$.

Clearly, if $\Theta$ is an equivalence relation, then $\widetilde{\Theta}$ is an equivalence relation, too. Additionally, if we consider the algebra $\left(F_{\mathcal{V}}(X), \Omega\right)$ and $\Theta$ is a congruence on $\left(\mathcal{P}_{>0}^{<\omega} F_{\mathcal{V}}(X), \Omega, \cup\right)$, then also $\widetilde{\Theta}$ is a congruence relation on $\left(F_{\mathcal{V}}(X), \Omega\right)$.

Lemma 3.7 Let $\Theta$ be a fully invariant congruence relation on $\left(\mathcal{P}_{>0}^{<\omega} F_{\mathcal{V}}(X), \Omega, \cup\right)$. Then the relation $\widetilde{\Theta}$ is a fully invariant congruence on $\left(F_{\mathcal{V}}(X), \Omega\right)$.

Proof Let $t, u \in F_{\mathcal{V}}(X)$. We have to prove that, if $(t, u) \in \widetilde{\Theta}$ then also $(\alpha(t), \alpha(u)) \in$ $\widetilde{\Theta}$, for any endomorphism $\alpha$ of the algebra $\left(F_{\mathcal{V}}(X), \Omega\right)$.

It was shown in [2] that if $\alpha: A \rightarrow B$ is a homomorphism of $\Omega$-algebras $(A, \Omega)$ and $(B, \Omega)$, so is $\alpha^{+}: \mathcal{P}_{>0}^{<\omega} A \rightarrow \mathcal{P}_{>0}^{<\omega} B$, where $\alpha^{+}(S):=\{\alpha(s) \mid s \in S\}$ for any $S \in$ $\mathcal{P}_{>0}^{<\omega} A$. Obviously, $\alpha^{+}$is also a homomorphism with respect to $\cup$. If $\alpha$ is an endomorphism of the algebra $\left(F_{\mathcal{V}}(X), \Omega\right)$, then $\alpha^{+}$is an endomorphism of the algebra $\left(\mathcal{P}_{>0}^{<\omega} F_{\mathcal{V}}(X), \Omega, \cup\right)$.

Since, by assumption, $\Theta$ is a fully invariant congruence on $\left(\mathcal{P}_{>0}^{<\omega} F_{\mathcal{V}}(X), \Omega, \cup\right)$, then in particular $(\{t\},\{u\}) \in \Theta$ implies $\left(\alpha^{+}(\{t\}), \alpha^{+}(\{u\})\right) \in \Theta$. Since $\alpha^{+}(\{x\})=\{\alpha(x)\}$ for any $x \in A$, one obtains $(\alpha(t), \alpha(u)) \in \widetilde{\Theta}$. 
Let $\operatorname{Con}_{f i}\left(F_{\mathcal{V}}(X)\right)$ be the set of all fully invariant congruences of the algebra $\left(F_{\mathcal{V}}(X), \Omega\right)$ and denote the set of all fully invariant congruences of the algebra $\left(\mathcal{P}_{>0}^{<\omega} F_{\mathcal{V}}(X), \Omega, \cup\right)$ by $\operatorname{Con}_{f i}\left(\mathcal{P}_{>0}^{<\omega} F_{\mathcal{V}}(X)\right)$.

From now through all over the paper, we assume $X$ is an infinite set. By Lemma 3.7, each fully invariant congruence relation $\Theta$ on $\left(\mathcal{P}_{>0}^{<\omega} F_{\mho}(X), \Omega, \cup\right)$ determines a subvariety $\mho_{\widetilde{\Theta}}$ of $\mho$ :

$$
\mho_{\widetilde{\Theta}}:=\operatorname{HSP}\left(\left(F_{\mho}(X) / \widetilde{\Theta}, \Omega\right)\right) .
$$

Of course, it may happen that for $\Theta_{1} \neq \Theta_{2}$, the varieties $\mho_{\widetilde{\Theta}_{1}}$ and $\mho_{\widetilde{\Theta}_{2}}$ are equal.

Example 3.8 By results of McKenzie and Romanowska [10] we have that there are at least five subvarieties of the variety of semilattice ordered groupoids which are also semilattice ordered semilattices. But the variety of semilattices has only two subvarieties.

We will show in Theorem 3.14 that for $\Theta_{1}, \Theta_{2} \in \operatorname{Con}_{f i}\left(\mathcal{P}_{>0}^{<\omega} F_{\mho}(X)\right)$, different congruences $\widetilde{\Theta}_{1} \neq \widetilde{\Theta}_{2} \in \operatorname{Con}_{f i}\left(F_{\mho}(X)\right)$ always determine different subvarieties of the variety $\mathcal{S}_{\mho}$. But first we prove some auxiliary results.

Lemma 3.9 Let $\Theta \in \operatorname{Con}_{f i}\left(\mathcal{P}_{>0}^{<\omega} F_{\mho}(X)\right)$ and $t=t\left(x_{1}, \ldots, x_{k}\right), u=u\left(x_{1}, \ldots, x_{k}\right) \in$ $F_{\mho}(X)$ be $k$-ary terms. Then, the identity $t \approx u$ holds in $\left(\mathcal{P}_{>0}^{<\omega} F_{\mho}(X) / \Theta, \Omega, \cup\right)$ if and only if $(\{t\},\{u\}) \in \Theta$.

Proof Let the identity $t \approx u$ hold in $\left(\mathcal{P}_{>0}^{<\omega} F_{\mho}(X) / \Theta, \Omega, \cup\right)$. This means that for any $P_{1} / \Theta, \ldots, P_{k} / \Theta \in \mathcal{P}_{>0}^{<\omega} F_{\mho}(X) / \Theta$, we have

$$
t\left(P_{1}, \ldots, P_{k}\right) / \Theta=t\left(P_{1} / \Theta, \ldots, P_{k} / \Theta\right)=u\left(P_{1} / \Theta, \ldots, P_{k} / \Theta\right)=u\left(P_{1}, \ldots, P_{k}\right) / \Theta .
$$

The latter implies that for any subsets $P_{1}, \ldots, P_{k} \in \mathcal{P}_{>0}^{<\omega} F_{\mho}(X)$, one has $\left(t\left(P_{1}, \ldots, P_{k}\right), u\left(P_{1}, \ldots, P_{k}\right)\right) \in \Theta$. In particular, for $P_{1}=\left\{x_{1}\right\}, \ldots, P_{k}=\left\{x_{k}\right\}$, we obtain $\left(\left\{t\left(x_{1}, \ldots, x_{k}\right)\right\},\left\{u\left(x_{1}, \ldots, x_{k}\right)\right\}\right) \in \Theta$.

Now, let $(\{t\},\{u\}) \in \Theta$. Since $\Theta \in \operatorname{Con}_{f i}\left(\mathcal{P}_{>0}^{<\omega} F_{\mho}(X)\right)$, then for any endomorphism $\alpha$ of $\left(\mathcal{P}_{>0}^{<\omega} F_{\mho}(X), \Omega, \cup\right)$ we also have $(\alpha(\{t\}), \alpha(\{u\})) \in \Theta$. In particular, for any subsets $P_{1}, \ldots, P_{k} \in \mathcal{P}_{>0}^{<\omega} F_{\mho}(X)$, we obtain $\left(t\left(P_{1}, \ldots, P_{k}\right), u\left(P_{1}, \ldots, P_{k}\right)\right) \in \Theta$. This means that the identity $t \approx u$ holds in $\left(\mathcal{P}_{>0}^{<\omega} F_{\mho}(X) / \Theta, \Omega, \cup\right)$.

Corollary 3.10 Let $\Theta \in \operatorname{Con}_{f i}\left(\mathcal{P}_{>0}^{<\omega} F_{\mho}(X)\right)$ and $t, u \in F_{\mho}(X)$. Then, the identity $t \approx u$ holds in $\left(\mathcal{P}_{>0}^{<\omega} F_{\mho}(X) / \Theta, \Omega\right)$ if and only if $t \approx u$ holds in $\left(F_{\mho}(X) / \widetilde{\Theta}, \Omega\right)$.

Corollary 3.11 Let $\Theta \in \operatorname{Con}_{f i}\left(\mathcal{P}_{>0}^{<\omega} F_{\mho}(X)\right)$. Then

$$
\operatorname{HSP}\left(\left(\mathcal{P}_{>0}^{<\omega} F_{\mho}(X) / \Theta, \Omega, \cup\right)\right) \subseteq \mathcal{S}_{\mho_{\tilde{\Theta}}} .
$$

Lemma 3.12 Let $\Theta, \Psi \in \operatorname{Con}_{f i}\left(\mathcal{P}_{>0}^{<\omega} F_{\mho}(X)\right)$ be such that $\left(\mathcal{P}_{>0}^{<\omega} F_{\mho}(X) / \Psi, \Omega, \cup\right) \in$ $\mathcal{S}_{\mho_{\widetilde{\Theta}}}$. Then, $\widetilde{\Theta} \subseteq \widetilde{\Psi}$.

Proof Let $t, u \in F_{\mho}(X)$ and $(t, u) \in \widetilde{\Theta}$. By assumption, $\left(\mathcal{P}_{>0}^{<\omega} F_{\mho}(X) / \Psi, \Omega\right) \in \mho_{\widetilde{\Theta}}$. This means the identity $t \approx u$ holds also in $\left(\mathcal{P}_{>0}^{<\omega} F_{\mho}(X) / \Psi, \Omega, \cup\right)$. By Lemma 3.9 we obtain $(t, u) \in \widetilde{\Psi}$ which shows $\widetilde{\Theta} \subseteq \widetilde{\Psi}$. 
Corollary 3.13 Let $\Theta, \Psi \in \operatorname{Con}_{f i}\left(\mathcal{P}_{>0}^{<\omega} F_{\mho}(X)\right)$ be such that

$$
\mathcal{S}_{\mho_{\tilde{\Theta}}}=\operatorname{HSP}\left(\left(\mathcal{P}_{>0}^{<\omega} F_{\mho}(X) / \Psi, \Omega, \cup\right)\right) \text {. }
$$

Then, $\widetilde{\Psi}=\widetilde{\Theta}$.

Proof By Lemma 3.12, we have $\widetilde{\Theta} \subseteq \widetilde{\Psi}$. Further, by Corollary 3.11 we also have $\operatorname{HSP}\left(\left(\mathcal{P}_{>0}^{<\omega} F_{\mho}(X) / \Theta, \Omega, \cup\right)\right) \subseteq \operatorname{HSP}\left(\left(\mathcal{P}_{>0}^{<\omega} F_{\mho}(X) / \Psi, \Omega, \cup\right)\right)$. Therefore, $\Psi \subseteq$ $\Theta$. Hence, $\widetilde{\Psi} \subseteq \widetilde{\Theta}$, which proves $\widetilde{\Psi}=\widetilde{\Theta}$.

By Corollary 3.11 and Lemma 3.12 one immediately obtains

Theorem 3.14 Let $\Theta_{1}, \Theta_{2} \in \operatorname{Con}_{f i}\left(\mathcal{P}_{>0}^{<\omega} F_{\mho}(X)\right)$. Then

$$
\widetilde{\Theta}_{1} \neq \widetilde{\Theta}_{2} \Rightarrow \mathcal{S}_{\mho_{\tilde{\Theta}_{1}}} \neq \mathcal{S}_{\mho_{\tilde{\Theta}_{2}}} \text {. }
$$

Finally note that for each subvariety $\mathcal{S}_{\mathcal{V}}$, with $\mathcal{V}$ the subvariety of $\mathcal{V}$, there exists a congruence $\Theta \in \operatorname{Con}_{f i}\left(\mathcal{P}_{>0}^{<\omega} F_{\mho}(X)\right)$ such that $\mathcal{V}=\mho_{\widetilde{\Theta}}$. Indeed, let

$$
\mathcal{S}_{\mathcal{V}}=\operatorname{HSP}\left(\left(\mathcal{P}_{>0}^{<\omega} F_{\mho}(X) / \Theta, \Omega, \cup\right)\right)
$$

and

$$
\mathcal{S}_{\mho_{\tilde{\Theta}}}=\operatorname{HSP}\left(\left(\mathcal{P}_{>0}^{<\omega} F_{\mho}(X) / \Psi, \Omega, \cup\right)\right),
$$

for some $\Theta, \Psi \in \operatorname{Con}_{f i}\left(\mathcal{P}_{>0}^{<\omega} F_{\mho}(X)\right)$. By Corollary 3.11, $\mathcal{S}_{\mathcal{V}} \subseteq \mathcal{S}_{\mho_{\tilde{\Theta}}}$. Further, by Corollary 3.13 we have $\widetilde{\Theta}=\widetilde{\Psi}$. Let $t, u \in F_{\mho}(X)$ and let the identity $t \approx u$ hold in $\mathcal{S}_{\mathcal{V}}$. By Corollary 3.10, we have that $t \approx u$ holds in $\mho_{\widetilde{\Theta}}=\mho_{\widetilde{\Psi}}$. This implies that $t \approx u$ holds in $\left(\mathcal{P}_{>0}^{<\omega} F_{\mho}(X) / \Psi, \Omega\right)$ and proves that $\mathcal{S}_{\mho_{\tilde{\Theta}}} \subseteq \mathcal{S}_{\mathcal{V}}$.

\section{4 "Main Knots" in the Lattice of Subvarieties}

In previous section we have shown that for different congruences $\widetilde{\Theta}_{1} \neq \widetilde{\Theta}_{2} \in$ $\operatorname{Con}_{f i}\left(F_{\mho}(X)\right)$, with $\Theta_{1}, \Theta_{2} \in \operatorname{Con}_{f i}\left(\mathcal{P}_{>0}^{<\omega} F_{\mho}(X)\right)$, the subvarieties $\mathcal{S}_{\mho_{\tilde{\Theta}_{1}}}$ and $\mathcal{S}_{\mho_{\tilde{\Theta}_{2}}}$ are always different. To describe all fully invariant congruences of the algebra $\left(\mathcal{P}_{>0}^{<\omega} F_{\mho}(X), \Omega, \cup\right)$ which uniquely determine subvarieties of the form $\mathcal{S}_{\mathcal{V}}$, for some $\mathcal{V} \subseteq \mathcal{V}$, we have to introduce the next binary relation.

Let us define a binary relation $\Re$ on the set $\operatorname{Con}_{f i}\left(\mathcal{P}_{>0}^{<\omega} F_{\mho}(X)\right)$ in the following way: for $\Theta_{1}, \Theta_{2} \in \operatorname{Con}_{f i}\left(\mathcal{P}_{>0}^{<\omega} F_{\mho}(X)\right)$

$$
\Theta_{1} \Re \Theta_{2} \Leftrightarrow \widetilde{\Theta}_{1}=\widetilde{\Theta}_{2} .
$$

Obviously, $\Re$ is an equivalence relation.

Theorem 4.1 Let $\Theta, \Psi \in \operatorname{Con}_{f i}\left(\mathcal{P}_{>0}^{<\omega} F_{\mho}(X)\right)$ be congruences such that $\mathcal{S}_{\mho_{\tilde{\Theta}}}=$ $\operatorname{HSP}\left(\left(\mathcal{P}_{>0}^{<\omega} F_{\mho}(X) / \Psi, \Omega, \cup\right)\right)$. Then, $\Psi=\bigcap_{\Phi \in \Theta / \Re} \Phi$.

Proof Let $\Theta, \Psi \in \operatorname{Con}_{f i}\left(\mathcal{P}_{>0}^{<\omega} F_{\mho}(X)\right)$ and $\mathcal{S}_{\mho_{\tilde{\Theta}}}=\operatorname{HSP}\left(\left(\mathcal{P}_{>0}^{<\omega} F_{\mho}(X) / \Psi, \Omega, \cup\right)\right)$. By Corollary 3.13 we have $\Psi \in \Theta / \Re$, thus obviously $\bigcap_{\Phi \in \Theta / \Re} \Phi \subseteq \Psi$.

On the other hand, for $\Phi \in \Theta / \Re$, we have $\widetilde{\Phi}=\widetilde{\Theta}$. Hence, by Corollary 3.11

$$
\operatorname{HSP}\left(\left(\mathcal{P}_{>0}^{<\omega} F_{\mho}(X) / \Phi, \Omega, \cup\right)\right) \subseteq \mathcal{S}_{\mho_{\tilde{\Phi}}}=\mathcal{S}_{\mho_{\tilde{\Theta}}}=\operatorname{HSP}\left(\left(\mathcal{P}_{>0}^{<\omega} F_{\mho}(X) / \Psi, \Omega, \cup\right)\right),
$$


which implies $\Psi \subseteq \bigcap_{\Phi \in \Theta / \Re} \Phi$, and consequently, $\Psi=\bigcap_{\Phi \in \Theta / \Re} \Phi$.

Corollary 4.2 Let $\Theta_{1}, \Theta_{2} \in \operatorname{Con}_{f i}\left(\mathcal{P}_{>0}^{<\omega} F_{\mho}(X)\right)$. Then

$$
\widetilde{\Theta}_{1} \subseteq \widetilde{\Theta}_{2} \Rightarrow \bigcap_{\Phi \in \Theta_{1} / \Re} \Phi \subseteq \bigcap_{\Phi \in \Theta_{2} / \Re} \Phi .
$$

Proof Let $\Theta_{1}, \Theta_{2}, \Psi_{1}, \Psi_{2} \in \operatorname{Con}_{f i}\left(\mathcal{P}_{>0}^{<\omega} F_{\mho}(X)\right)$ be such that

$$
\begin{aligned}
& \mathcal{S}_{\mho_{\widetilde{\Theta}_{1}}}=\operatorname{HSP}\left(\left(\mathcal{P}_{>0}^{<\omega} F_{\mho}(X) / \Psi_{1}, \Omega, \cup\right)\right), \text { and } \\
& \mathcal{S}_{\mho_{\widetilde{\Theta}_{2}}}=\operatorname{HSP}\left(\left(\mathcal{P}_{>0}^{<\omega} F_{\mho}(X) / \Psi_{2}, \Omega, \cup\right)\right) .
\end{aligned}
$$

Since, by assumption, $\widetilde{\Theta}_{1} \subseteq \widetilde{\Theta}_{2}$, then by Corollaries 3.10 and 3.13 we have $\mathcal{S}_{\mho_{\widetilde{\Theta}_{2}}} \subseteq$ $\mathcal{S}_{\mho_{\tilde{\Theta}_{1}}}$.

By Theorem 4.1, this is clear that

$$
\bigcap_{\Phi \in \Theta_{1} / \Re} \Phi=\Psi_{1} \subseteq \Psi_{2}=\bigcap_{\Phi \in \Theta_{2} / \Re} \Phi
$$

which finishes the proof.

Lemma 4.3 Let $I$ be a set and for each $i \in I$, let $\Theta_{i}$ be an equivalence relation on $\mathcal{P}_{>0}^{<\omega} F_{\mho}(X)$. Then

$$
\bigcap_{i \in I} \widetilde{\Theta}_{i}=\widetilde{\bigcap_{i \in I} \Theta_{i}}
$$

Let $\Theta \in \operatorname{Con}_{f i}\left(\mathcal{P}_{>0}^{<\omega} F_{\mho}(X)\right), I$ be a set and for each $i \in I$, let $\Psi_{i} \in \Theta / \Re$. Then by Lemma 4.3, also $\bigcap_{i \in I} \Psi_{i} \in \Theta / \Re$. This shows that in each class of the relation $\Re$ there is the least element with respect to the set inclusion. Let

$$
\operatorname{Con}_{f i}^{\Re}\left(\mathcal{P}_{>0}^{<\omega} F_{\mho}(X)\right):=\left\{\Theta \in \operatorname{Con}_{f i}\left(\mathcal{P}_{>0}^{<\omega} F_{\mho}(X)\right) \mid \Theta=\bigcap_{\Phi \in \Theta / \Re} \Phi\right\}
$$

be the set of all such the least congruences in each $\Re$-class.

Directly by Theorem 4.1 we obtain

Corollary 4.4 Let $\Theta \in \operatorname{Con}_{f i}^{\Re}\left(\mathcal{P}_{>0}^{<\omega} F_{\mho}(X)\right)$. Then

$$
\mathcal{S}_{\mho_{\tilde{\Theta}}}=\operatorname{HSP}\left(\left(\mathcal{P}_{>0}^{<\omega} F_{\mho}(X) / \Theta, \Omega, \cup\right)\right) .
$$

We can say that subvarieties of the form $\mathcal{S}_{\mho_{\tilde{\Theta}}}, \Theta \in \operatorname{Con}_{f i}^{\Re}\left(\mathcal{P}_{>0}^{<\omega} F_{\mho}(X)\right)$, are some kinds of "main knots" in the lattice of subvarieties of semilattice ordered algebras.

Obviously, if $\Theta_{1} \subseteq \Theta_{2}$ then also $\widetilde{\Theta}_{1} \subseteq \widetilde{\Theta}_{2}$. As a consequence of Corollary 4.2 if $\Theta_{1}, \Theta_{2} \in \operatorname{Con}_{f i}^{\Re}\left(\mathcal{P}_{>0}^{<\omega} F_{\mho}(X)\right)$, then the converse is also true. 
Corollary 4.5 Let $\Theta_{1}, \Theta_{2} \in \operatorname{Con}_{f i}^{\Re}\left(\mathcal{P}_{>0}^{<\omega} F_{\mho}(X)\right)$. Then

$$
\Theta_{1} \subseteq \Theta_{2} \Leftrightarrow \widetilde{\Theta}_{1} \subseteq \widetilde{\Theta}_{2} \text {. }
$$

Let $\Theta_{1} \vee \Theta_{2}$ denote the least upper bound of two congruence relations $\Theta_{1}, \Theta_{2} \in$ $\operatorname{Con}_{f i}\left(\mathcal{P}_{>0}^{<\omega} F_{\mho}(X)\right)$ with respect to the set inclusion. Note that in general $\widetilde{\Theta}_{1} \vee \widetilde{\Theta}_{2} \subsetneq$ $\widetilde{\Theta_{1} \vee \Theta_{2}}$.

Corollary 4.5 allows us to formulate the following result.

Lemma 4.6 The ordered set $\left(\operatorname{Con}_{f i}^{\Re}\left(\mathcal{P}_{>0}^{<\omega} F_{\mho}(X)\right), \subseteq\right)$ is a complete lattice. Let I be a set and for each $i \in I$, let $\Theta_{i} \in \operatorname{Con}_{f i}^{\Re}\left(\mathcal{P}_{>0}^{<\omega} F_{\mho}(X)\right)$. Then, the relation

$$
\bigcap_{\Phi \in\left(\bigvee_{i \in I} \Theta_{i}\right) / \Re} \Phi
$$

is the least upper bound of $\left\{\Theta_{i}\right\}_{i \in I}$, and the congruence

$$
\bigcap_{\Phi \in\left(\bigcap_{i \in I} \Theta_{i}\right) / \Re} \Phi
$$

is the greatest lower bound of $\left\{\Theta_{i}\right\}_{i \in I}$.

Let us introduce the following notation:

$$
\mathcal{L}^{\Re}(\mho):=\left\{\mho_{\widetilde{\Theta}} \subseteq \mho \mid \Theta \in \operatorname{Con}_{f i}^{\Re}\left(\mathcal{P}_{>0}^{<\omega} F_{\mho}(X)\right)\right\} .
$$

Clearly, the set $\mathcal{L}^{\Re}(\mho)$ is partially ordered by the set inclusion. Moreover, for $\Theta_{1}, \Theta_{2} \in \operatorname{Con}_{f i}^{\Re}\left(\mathcal{P}_{>0}^{<\omega} F_{\mho}(X)\right)$

$$
\mho_{\widetilde{\Theta}_{1}} \subseteq \mho_{\widetilde{\Theta}_{2}} \Leftrightarrow \widetilde{\Theta}_{2} \subseteq \widetilde{\Theta}_{1} \Leftrightarrow \Theta_{2} \subseteq \Theta_{1}
$$

Directly from Lemma 4.6 we obtain the following

Theorem 4.7 The ordered set $\left(\mathcal{L}^{\Re}(\mho), \subseteq\right)$ is a complete lattice dually isomorphic to the lattice $\left(\operatorname{Con}_{f i}^{\Re}\left(\mathcal{P}_{>0}^{<\omega} F_{\mho}(X)\right), \subseteq\right)$. For any two subvarieties $\mho_{\widetilde{\Theta}_{1}}, \mho_{\widetilde{\Theta}_{2}} \in \mathcal{L}^{\Re}(\mho)$, the variety $\mho_{\widetilde{\Theta_{1} \cap \Theta_{2}}}$ is the least upper bound of $\mho_{\widetilde{\Theta}_{1}}$ and $\mho_{\widetilde{\Theta}_{2}}$, and the variety $\mho_{\widetilde{\Theta_{1} \vee \Theta_{2}}}$ is the greatest lower bound of $\mho_{\widetilde{\Theta}_{1}}$ and $\mho_{\widetilde{\Theta}_{2}}$.

By Lemma 4.3 it follows that the variety $\mho_{\widetilde{\Theta_{1} \cap \Theta_{2}}}$ is equal to the variety $\mho_{\widetilde{\Theta}_{1}} \vee \mho_{\widetilde{\Theta}_{2}}$, the least upper bound of $\mho_{\widetilde{\Theta}_{1}}$ and $\mho_{\widetilde{\Theta}_{2}}$ with respect to the set inclusion. But the variety $\mho_{\widetilde{\Theta_{1} \vee \Theta_{2}}}$ has not to be equal to the variety $\mho_{\widetilde{\Theta}_{1}} \cap \mho_{\widetilde{\Theta}_{2}}$.

By Theorem 3.14 we immediately obtain

Corollary 4.8 The lattice $\left(\left\{\mathcal{S}_{\mho_{\tilde{\Theta}}} \mid \Theta \in \operatorname{Con}_{f i}^{\Re}\left(\mathcal{P}_{>0}^{<\omega} F_{\mho}(X)\right)\right\}, \subseteq\right)$ of all "main knots" subvarieties is isomorphic to the lattice $\left(\mathcal{L}^{\Re}(\mho), \subseteq\right)$. For any two varieties $\mathcal{S}_{\mho_{\widetilde{\Theta}_{1}}}$ and $\mathcal{S}_{\mho_{\widetilde{\Theta}_{2}}}$, the variety $\mathcal{S}_{\mho_{\widetilde{\Theta}_{1}} \vee \mho_{\widetilde{\Theta}_{2}}}$ is their least upper bound and the variety $\mathcal{S}_{\mho_{{\Theta_{1} \vee \Theta_{2}}}}$ is their greatest lower bound (see Fig. 1). 
Fig. 1 The lattice $\left(\left\{\mathcal{S}_{\mho_{\widetilde{\Theta}}} \mid \Theta \in\right.\right.$ $\left.\left.\operatorname{Con}_{f i}^{\Re}\left(\mathcal{P}_{>0}^{<\omega} F_{\mho}(X)\right)\right\}, \subseteq\right)$

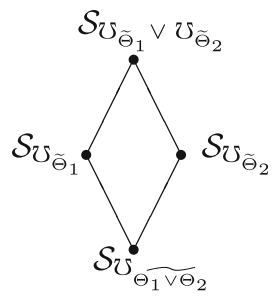

\section{The Lattice of Subvarieties}

By Lemma 4.3 one obtains the following characterization of the ordered set $(\Theta / \Re, \subseteq)$ for $\Theta \in \operatorname{Con}_{f i}^{\Re}\left(\mathcal{P}_{>0}^{<\omega} F_{\mho}(X)\right)$.

Lemma 5.1 Let $\Theta \in \operatorname{Con}_{f i}^{\Re}\left(\mathcal{P}_{>0}^{<\omega} F_{\mho}(X)\right)$, I be a set and for each $i \in I, \Psi_{i} \in \Theta / \Re$. Then $(\Theta / \Re, \subseteq)$ is a complete meet-semilattice with the relation $\bigcap_{i \in I} \Psi_{i}$ as the greatest lower bound of $\left\{\Psi_{i}\right\}_{i \in I}$.

Let $\Psi \in \Theta / \Re$. Note that by Corollary 3.10 the algebras $\left(\mathcal{P}_{>0}^{<\omega} F_{\mho}(X) / \Psi, \Omega\right)$ and $\left(F_{\mho}(X) / \widetilde{\Theta}, \Omega\right)$ satisfy exactly the same identities. This shows that the variety

$$
\mathcal{S}=\operatorname{HSP}\left(\left(\mathcal{P}_{>0}^{<\omega} F_{\mho}(X) / \Psi, \Omega, \cup\right)\right)
$$

is a subvariety of $\mathcal{S}_{\mho_{\tilde{\Theta}}}$ and is not included in $\mathcal{S}_{\mathcal{W}}$ for any proper subvariety $\mathcal{W} \subset \mho_{\widetilde{\Theta}}$. This justifies the introduction of the following definition.

Definition 5.2 Let $\mathcal{V}$ be a subvariety of $\mho$ and $\mathcal{S}$ be a non-trivial subvariety of $\mathcal{S}_{\mathcal{V}}$. The variety $\mathcal{S}$ is $\mathcal{V}$-preserved, if $\mathcal{S} \nsubseteq \mathcal{S}_{\mathcal{W}}$ for any proper subvariety $\mathcal{W}$ of $\mathcal{V}$.

Lemma 5.3 Let $\Theta, \Psi \in \operatorname{Con}_{f i}\left(\mathcal{P}_{>0}^{<\omega} F_{\mho}(X)\right)$. A non-trivial subvariety

$$
\mathcal{S}=\operatorname{HSP}\left(\left(\mathcal{P}_{>0}^{<\omega} F_{\mho}(X) / \Psi, \Omega, \cup\right)\right) \subseteq \mathcal{S}_{\mho_{\tilde{\Theta}}}
$$

is $\mho_{\widetilde{\Theta}}$-preserved if and only if $\widetilde{\Psi}=\widetilde{\Theta}$.

Proof By Lemma 3.12, for each subvariety $\mathcal{S}=\operatorname{HSP}\left(\left(\mathcal{P}_{>0}^{<\omega} F_{\mho}(X) / \Psi, \Omega, \cup\right)\right) \subseteq \mathcal{S}_{\mho_{\tilde{\Theta}}}$, one has $\widetilde{\Theta} \subseteq \widetilde{\Psi}$. Now let $\mathcal{S}$ be $\mho_{\widetilde{\Theta}}$-preserved subvariety of $\mathcal{S}_{\mho_{\widetilde{\Theta}}}$. By definition, $\mathcal{S}$ is not included in any variety $\mathcal{S}_{\mathcal{W}}$ for a proper subvariety $\mathcal{W} \subset \mho_{\widetilde{\Theta}}$. This means that the algebra $\left(\mathcal{P}_{>0}^{<\omega} F_{\mho}(X) / \Psi, \Omega\right)$ does not belong to any proper subvariety of $\mho_{\widetilde{\Theta}}$. Then, by Corollary 3.10, $\widetilde{\Psi}=\widetilde{\Theta}$.

Finally, let $\widetilde{\Psi}=\widetilde{\Theta}$. Hence, by Corollary 3.10 the algebras $\left(\mathcal{P}_{>0}^{<\omega} F_{\mho}(X) / \Psi, \Omega\right)$ and $\left(F_{\mho}(X) / \widetilde{\Theta}, \Omega\right)$ satisfy exactly the same identities, which shows that $\mathcal{S}$ is $\mho_{\widetilde{\Theta}^{-}}$ preserved.

Let $\Theta \in \operatorname{Con}_{f i}^{\Re}\left(\mathcal{P}_{>0}^{<\omega} F_{\mho}(X)\right)$. By Lemma 5.3 with each congruence $\Psi \in \Theta / \Re$ one can associate the $\mho_{\widetilde{\Theta}}$-preserved subvariety $\operatorname{HSP}\left(\left(\mathcal{P}_{>0}^{<\omega} F_{\mho}(X) / \Psi, \Omega, \cup\right)\right)$ of $\mathcal{S}_{\mho_{\tilde{\Theta}}}$. This mapping is the restriction of the dual isomorphism we have between lattice $\left(\operatorname{Con}_{f i}\left(\mathcal{P}_{>0}^{<\omega} F_{\mho}(X)\right), \subseteq\right)$ and the lattice of all subvarieties of $\mathcal{S}_{\mho}$, to the set $\Theta / \Re$. By Lemmas 4.3, 5.1 and 5.3 we immediately have 
Theorem 5.4 Let $\Theta \in \operatorname{Con}_{f i}^{\Re}\left(\mathcal{P}_{>0}^{<\omega} F_{\mho}(X)\right)$. The semilattice of all $\mho_{\widetilde{\Theta}}$-preserved subvarieties of $\mathcal{S}_{\mho_{\tilde{\Theta}}}$ is dually isomorphic to the complete semilattice $(\Theta / \Re, \subseteq)$. For any $\mho_{\widetilde{\Theta}}$-preserved subvarieties $\left\{\mathcal{S}_{i}\right\}_{i \in I}$ of $\mathcal{S}_{\mho_{\tilde{\Theta}}}$, the variety $\bigvee_{i \in I} \mathcal{S}_{i}$ is their least upper bound.

In Theorem 5.9 we will show that there is also a correspondence between the set of $\mho_{\widetilde{\Theta}}$-preserved subvarieties of $\mathcal{S}_{\mho_{\widetilde{\Theta}}}$ and the set of some fully invariant congruence relations on the algebra $\left(\mathcal{P}_{>0}^{<\omega}\left(F_{\mho}(X) / \widetilde{\Theta}\right), \Omega, \cup\right)$. First we will prove some auxiliary technical results.

We introduce the following notation. For a set $Q \in \mathcal{P}_{>0}^{<\omega} F_{\mho}(X)$ and a congruence $\Psi \in \operatorname{Con}_{f i}\left(\mathcal{P}_{>0}^{<\omega} F_{\mho}(X)\right)$,

$$
Q^{\widetilde{\Psi}}:=\left\{q\left(x_{1} / \widetilde{\Psi}, \ldots, x_{n} / \widetilde{\Psi}\right) \mid q \in Q\right\}=\left\{q\left(x_{1}, \ldots, x_{n}\right) / \widetilde{\Psi} \mid q \in Q\right\} \in \mathcal{P}_{>0}^{<\omega}\left(F_{\mho}(X) / \widetilde{\Psi}\right) .
$$

Now, we define a relation $\delta_{\Psi} \subseteq \mathcal{P}_{>0}^{<\omega}\left(F_{\mho}(X) / \widetilde{\Psi}\right) \times \mathcal{P}_{>0}^{<\omega}\left(F_{\mho}(X) / \widetilde{\Psi}\right)$ in the following way:

$$
\left(Q^{\widetilde{\Psi}}, R^{\widetilde{\Psi}}\right) \in \delta_{\Psi} \quad \Leftrightarrow \quad(Q, R) \in \Psi
$$

for $Q, R \in \mathcal{P}_{>0}^{<\omega} F_{\mho}(X)$.

Lemma 5.5 Let $\Psi \in \operatorname{Con}_{f i}\left(\mathcal{P}_{>0}^{<\omega} F_{\mho}(X)\right)$. The relation $\delta_{\Psi}$ is a fully invariant congruence relation on $\left(\mathcal{P}_{>0}^{<\omega}\left(F_{\mho}(X) / \widetilde{\Psi}\right), \Omega, \cup\right)$ such that $\widetilde{\delta}_{\Psi}=i d_{F_{\mho}(X) / \widetilde{\Psi}}$ and $\left(\mathcal{P}_{>0}^{<\omega}\left(F_{\mho}(X) / \widetilde{\Psi}\right) / \delta_{\Psi}, \Omega\right) \in \mho_{\widetilde{\Psi}}$.

Proof Let $\Psi \in \operatorname{Con}_{f i}\left(\mathcal{P}_{>0}^{<\omega} F_{\mho}(X)\right)$. First note that the definition of the relation $\delta_{\Psi}$ is correct. Let $x_{1}, \ldots, x_{k}, y_{1}, \ldots, y_{m} \in F_{\mho}(X)$ and $\left(x_{1}, t_{1}\right), \ldots,\left(x_{k}, t_{k}\right),\left(y_{1}, u_{1}\right), \ldots$, $\left(y_{m}, u_{m}\right) \in \widetilde{\Psi}$. Then $\left(\left\{x_{1}\right\},\left\{t_{1}\right\}\right), \ldots,\left(\left\{x_{k}\right\},\left\{t_{k}\right\}\right),\left(\left\{y_{1}\right\},\left\{u_{1}\right\}\right), \ldots,\left(\left\{y_{m}\right\},\left\{u_{m}\right\}\right) \in \Psi$. Since $\Psi$ is a congruence on $\left(\mathcal{P}_{>0}^{<\omega} F_{\mho}(X), \Omega, \cup\right)$ we obtain

$$
\left(\left\{x_{1}, \ldots, x_{k}\right\},\left\{t_{1}, \ldots, t_{k}\right\}\right),\left(\left\{y_{1}, \ldots, y_{m}\right\},\left\{u_{1}, \ldots, u_{m}\right\}\right) \in \Psi .
$$

Clearly, the relation $\delta_{\Psi}$ is a congruence on $\left(\mathcal{P}_{>0}^{<\omega}\left(F_{\mho}(X) / \widetilde{\Psi}\right), \Omega, \cup\right)$. We will show that $\delta_{\Psi}$ is fully invariant. Let $Q^{\widetilde{\Psi}}, R^{\widetilde{\Psi}} \in \mathcal{P}_{>0}^{<\omega}\left(F_{\mho}(X) / \widetilde{\Psi}\right)$. We have to prove that for every endomorphism $\lambda$ of $\left(\mathcal{P}_{>0}^{<\omega}\left(F_{\mho}(X) / \widetilde{\Psi}\right), \Omega, \cup\right)$, if $\left(Q^{\widetilde{\Psi}}, R^{\widetilde{\Psi}}\right) \in \delta_{\Psi}$, then also $\left(\lambda\left(Q^{\widetilde{\Psi}}\right), \lambda\left(R^{\widetilde{\Psi}}\right)\right) \in \delta_{\Psi}$.

For each $x_{i} / \widetilde{\Psi} \in X / \widetilde{\Psi}$ let us choose a subset $P_{i}^{\widetilde{\Psi}} \in \mathcal{P}_{>0}^{<\omega}\left(F_{\mho}(X) / \widetilde{\Psi}\right)$. The mapping $\lambda: \mathcal{P}_{>0}^{<\omega}\left(F_{\mho}(X) / \widetilde{\Psi}\right) \rightarrow \mathcal{P}_{>0}^{<\omega}\left(F_{\mho}(X) / \widetilde{\Psi}\right)$

$$
\lambda\left(Q^{\widetilde{\Psi}}\right)=\lambda\left(\left\{q\left(x_{1} / \widetilde{\Psi}, \ldots, x_{n} / \widetilde{\Psi}\right) \mid q \in Q\right\}\right):=\bigcup_{q \in Q} q\left(P_{1}^{\widetilde{\Psi}}, \ldots, P_{n}^{\widetilde{\Psi}}\right)
$$

is an endomorphism of the algebra $\left(\mathcal{P}_{>0}^{<\omega}\left(F_{\mho}(X) / \widetilde{\Psi}\right), \Omega, \cup\right)$.

Note that the algebra $\left(F_{\mho}(X) / \widetilde{\Psi}, \Omega\right)$ is generated by the set $\{x / \widetilde{\Psi} \mid x \in X\}$. It follows then that the algebra $\left(\mathcal{P}_{>0}^{<\omega}\left(F_{\mho}(X) / \widetilde{\Psi}\right), \Omega, \cup\right)$ is generated by the set $\{\{x / \widetilde{\Psi}\} \mid$ $x \in X\}$ and for any $\left\{x_{i} / \widetilde{\Psi}\right\}$, we have $\lambda\left(\left\{x_{i} / \widetilde{\Psi}\right\}\right)=P_{i}^{\widetilde{\Psi}}$. Because each homomorphism is uniquely defined on generators of an algebra then we obtain that each endomorphism $\lambda$ of $\left(\mathcal{P}_{>0}^{<\omega}\left(F_{\mho}(X) / \widetilde{\Psi}\right), \Omega, \cup\right)$ is of the form Eq. 5.1.

Recall we consider here only finite subsets of $F_{\mho}(X)$. This assumption is crucial in what follows. Let $\Lambda$ be an endomorphism of $\left(\mathcal{P}_{>0}^{<\omega} F_{\mho}(X), \Omega, \cup\right)$. By above discussion, 
$\Lambda$ is of the form Eq. 5.1 for subsets $P_{1}, \ldots, P_{n} \in \mathcal{P}_{>0}^{<\omega} F_{\mho}(X)$. Let $Q, R \in \mathcal{P}_{>0}^{<\omega} F_{\mho}(X)$. Hence we have

$$
\left(Q^{\widetilde{\Psi}}, R^{\widetilde{\Psi}}\right) \in \delta_{\Psi} \quad \Leftrightarrow(Q, R) \in \Psi .
$$

Therefore, because $\Psi$ is, by assumption, a fully invariant congruence on $\left(\mathcal{P}_{>0}^{<\omega} F_{\mho}(X), \Omega, \cup\right)$, for the endomorphism $\Lambda$ we obtain

$$
\begin{aligned}
& (\Lambda(Q), \Lambda(R)) \in \Psi \Rightarrow\left(\bigcup_{q \in Q} q\left(P_{1}, \ldots, P_{n}\right), \bigcup_{r \in R} r\left(P_{1}, \ldots, P_{n}\right)\right) \in \Psi \Rightarrow \\
& \left(\left(\bigcup_{q \in Q} q\left(P_{1}, \ldots, P_{n}\right)\right)^{\widetilde{\Psi}},\left(\bigcup_{r \in R} r\left(P_{1}, \ldots, P_{n}\right)\right)^{\widetilde{\Psi}}\right) \in \delta_{\Psi} \Rightarrow \\
& \left(\bigcup_{q \in Q} q\left(P_{1}^{\widetilde{\Psi}}, \ldots, P_{n}^{\widetilde{\Psi}}\right), \bigcup_{r \in R} r\left(P_{1}^{\widetilde{\Psi}}, \ldots, P_{n}^{\widetilde{\Psi}}\right)\right) \in \delta_{\Psi} .
\end{aligned}
$$

This shows that for any endomorphism $\Upsilon$ of the algebra $\left(\mathcal{P}_{>0}^{<\omega}\left(F_{\mho}(X) / \widetilde{\Psi}\right), \Omega, \cup\right)$, $\left(\Upsilon\left(Q^{\widetilde{\Psi}}\right), \Upsilon\left(R^{\widetilde{\Psi}}\right)\right) \in \delta_{\Psi}$ and proves that the congruence $\delta_{\Psi}$ is fully invariant.

Now we will prove that $\left(\mathcal{P}_{>0}^{<\omega}\left(F_{\mho}(X) / \widetilde{\Psi}\right) / \delta_{\Psi}, \Omega\right) \in \mho_{\widetilde{\Psi}}$. By Corollary 3.11, the variety $\operatorname{HSP}\left(\left(\mathcal{P}_{>0}^{<\omega} F_{\mho}(X) / \Psi, \Omega, \cup\right)\right)$ is included in $\mathcal{S}_{\mho_{\tilde{\Psi}}}$, which means that the algebra $\left(\mathcal{P}_{>0}^{<\omega} F_{\mho}(X) / \Psi, \Omega\right)$ belongs to the variety $\mho_{\widetilde{\Psi}}$. Hence, for any identity $t\left(x_{1}, \ldots, x_{m}\right)=t \approx u=u\left(x_{1}, \ldots, x_{m}\right)$ which holds in $\mho_{\widetilde{\Psi}}$ and arbitrary $Q_{1} / \Psi, \ldots, Q_{m} / \Psi \in \mathcal{P}_{>0}^{<\omega} F_{\mho}(X) / \Psi$ we have

$$
\begin{aligned}
& t\left(Q_{1}, \ldots, Q_{m}\right) / \Psi=t\left(Q_{1} / \Psi, \ldots, Q_{m} / \Psi\right)= \\
& u\left(Q_{1} / \Psi, \ldots, Q_{m} / \Psi\right)=u\left(Q_{1}, \ldots, Q_{m}\right) / \Psi
\end{aligned}
$$

This is equivalent to

$$
\left(t\left(Q_{1}, \ldots, Q_{m}\right), u\left(Q_{1}, \ldots, Q_{m}\right)\right) \in \Psi
$$

It follows then

$$
\begin{aligned}
& \left(t\left(Q_{1}, \ldots, Q_{m}\right)^{\widetilde{\Psi}}, u\left(Q_{1}, \ldots, Q_{m}\right)^{\widetilde{\Psi}}\right) \in \delta_{\Psi} \Rightarrow \\
& \left(t\left(Q_{1}^{\widetilde{\Psi}}, \ldots, Q_{m}^{\widetilde{\Psi}}\right), u\left(Q_{1}^{\widetilde{\Psi}}, \ldots, Q_{m}^{\widetilde{\Psi}}\right)\right) \in \delta_{\Psi} \Leftrightarrow \\
& t\left(Q_{1}^{\widetilde{\Psi}}, \ldots, Q_{m}^{\widetilde{\Psi}}\right) / \delta_{\Psi}=u\left(Q_{1}^{\widetilde{\Psi}}, \ldots, Q_{m}^{\widetilde{\Psi}}\right) / \delta_{\Psi} \Leftrightarrow \\
& t\left(Q_{1}^{\widetilde{\Psi}} / \delta_{\Psi}, \ldots, Q_{m}^{\widetilde{\Psi}} / \delta_{\Psi}\right)=u\left(Q_{1}^{\widetilde{\Psi}} / \delta_{\Psi}, \ldots, Q_{m}^{\widetilde{\Psi}} / \delta_{\Psi}\right) .
\end{aligned}
$$

This proves that the identity $t \approx u$ holds in the algebra $\left(\mathcal{P}_{>0}^{<\omega}\left(F_{\mho}(X) / \widetilde{\Psi}\right) / \delta_{\Psi}, \Omega\right)$.

Finally, for $t, u \in F_{\mho}(X)$

$$
\begin{aligned}
& (t / \widetilde{\Psi}, u / \widetilde{\Psi}) \in \widetilde{\delta}_{\Psi} \Leftrightarrow(\{t / \widetilde{\Psi}\},\{u / \widetilde{\Psi}\}) \in \delta_{\Psi} \Leftrightarrow(\{t\},\{u\}) \in \Psi \Leftrightarrow \\
& (t, u) \in \widetilde{\Psi} \Leftrightarrow t / \widetilde{\Psi}=u / \widetilde{\Psi} \Leftrightarrow(t / \widetilde{\Psi}, u / \widetilde{\Psi}) \in i d_{F_{\mho}(X) / \widetilde{\Psi}}
\end{aligned}
$$


Let $\Theta \in \operatorname{Con}_{f i}^{\Re}\left(\mathcal{P}_{>0}^{<\omega} F_{\mho}(X)\right)$. Denote by $\operatorname{Con}_{f i}^{i d}\left(\mathcal{P}_{>0}^{<\omega} F_{\mho_{\Theta}}(X)\right)$ the following set:

$$
\left\{\psi \in \operatorname{Con}_{f i}\left(\mathcal{P}_{>0}^{<\omega} F_{\mho_{\tilde{\Theta}}}(X)\right) \mid \tilde{\psi}=i d_{F_{\widetilde{\Theta}}(X)} \text { and }\left(\mathcal{P}_{>0}^{<\omega} F_{\mho_{\tilde{\Theta}}}(X) / \psi, \Omega\right) \in \mho_{\widetilde{\Theta}}\right\} .
$$

Now, for $\psi \in \operatorname{Con}_{f i}^{i d}\left(\mathcal{P}_{>0}^{<\omega} F_{\mho}(X)\right)$, define a relation $\Delta_{\psi} \subseteq \mathcal{P}_{>0}^{<\omega} F_{\mho}(X) \times \mathcal{P}_{>0}^{<\omega} F_{\mho}(X)$ in the following way:

$$
(T, U) \in \Delta_{\psi} \quad \Leftrightarrow \quad\left(T^{\widetilde{\Theta}}, U^{\widetilde{\Theta}}\right) \in \psi .
$$

Again, by similar straightforward calculations as in the proof of Lemma 5.5 one can obtain the following.

Lemma 5.6 Let $\Theta \in \operatorname{Con}_{f i}^{\Re}\left(\mathcal{P}_{>0}^{<\omega} F_{\mho}(X)\right)$ and $\psi \in \operatorname{Con}_{f i}^{i d}\left(\mathcal{P}_{>0}^{<\omega} F_{\mho_{\tilde{\Theta}}}(X)\right)$. Then, the relation $\Delta_{\psi}$ is a fully invariant congruence on $\left(\mathcal{P}_{>0}^{<\omega} F_{\mho}(X), \Omega, \cup\right)$ with the properties $\widetilde{\Delta}_{\psi}=\widetilde{\Theta}$ and $\operatorname{HSP}\left(\left(\mathcal{P}_{>0}^{<\omega} F_{\mho}(X) / \Delta_{\psi}, \Omega\right)\right) \subseteq \mho_{\widetilde{\Theta}}$.

Lemma 5.7 Let $\Theta \in \operatorname{Con}_{f i}^{\Re}\left(\mathcal{P}_{>0}^{<\omega} F_{\mho}(X)\right)$.

If $\Psi \in \Theta / \Re$ then

$$
\Psi=\Delta_{\delta_{\Psi}} .
$$

For a congruence $\psi \in \operatorname{Con}_{f i}^{i d}\left(\mathcal{P}_{>0}^{<\omega} F_{\mho_{\tilde{\Theta}}}(X)\right)$ one has

$$
\psi=\delta_{\Delta_{\psi}} .
$$

Lemmas 5.5 and 5.7 immediately imply

Corollary 5.8 Let $\Theta \in \operatorname{Con}_{f i}^{\Re}\left(\mathcal{P}_{>0}^{<\omega} F_{\mho}(X)\right)$. The map $\Psi \mapsto \delta_{\Psi}$ is a complete semilattice homomorphism between $(\Theta / \Re, \subseteq)$ and $\left(\operatorname{Con}_{f i}^{i d}\left(\mathcal{P}_{>0}^{<\omega} F_{\mho_{\tilde{\Theta}}}(X)\right), \subseteq\right)$.

By Corollary 5.8 and Theorem 5.4 we obtain

Theorem 5.9 Let $\Theta \in \operatorname{Con}_{f i}^{\Re}\left(\mathcal{P}_{>0}^{<\omega} F_{\mho}(X)\right)$. The complete semilattice of all $\mho_{\widetilde{\Theta}^{-}}$ preserved subvarieties of $\mathcal{S}_{\mho_{\tilde{\Theta}}}$ is dually isomorphic to the complete semilattice $\left(\operatorname{Con}_{f i}^{i d}\left(\mathcal{P}_{>0}^{<\omega} F_{\mho_{\tilde{\Theta}}}(X)\right), \subseteq\right)$.

Before we formulate Theorem 5.12, the main result of the paper, let us summarize what we have already known. For each $\Psi \in \operatorname{Con}_{f i}\left(\mathcal{P}_{>0}^{<\omega} F_{\mho}(X)\right)$ such that $\widetilde{\Psi}=\widetilde{\Theta}$, the subvariety $\mathcal{S}=\operatorname{HSP}\left(\left(\mathcal{P}_{>0}^{<\omega} \mathrm{F}_{\mho}(\mathrm{X}) / \Psi, \Omega, \cup\right)\right)$ of $\mathcal{S}_{\mho}$ is an $\mho_{\widetilde{\Theta}}$-preserved subvariety of $\mathcal{S}_{\mho_{\tilde{\Theta}}} \subseteq \mathcal{S}_{\mho}$ according to Lemma 5.3. Therefore, to find any subvariety $\mathcal{S}$ of $\mathcal{S}_{\mho}$ we should proceed as follows: first find the proper "main knot" and then choose one of its subvarieties.

By Theorem 3.14, Lemma 5.3, and Theorem 5.9 one can uniquely associate with the variety $\mathcal{S}$ two congruence relations: $\Theta \in \operatorname{Con}_{f i}^{\Re}\left(\mathcal{P}_{>0}^{<\omega} F_{\mho}(X)\right)$ such that $\widetilde{\Psi}=\widetilde{\Theta}$ (for the "main knot" $\mathcal{S}_{\mho_{\tilde{\Theta}}}$ ), and then $\delta^{\Psi} \in \operatorname{Con}_{f i}^{i d}\left(\mathcal{P}_{>0}^{<\omega} F_{\mho_{\tilde{\Theta}}}(X)\right.$ ) (for the chosen $\mho_{\widetilde{\Theta}^{-}}$ preserved subvariety).

On the other hand, any pair of congruences: $\Theta \in \operatorname{Con}_{f i}^{\Re i}\left(\mathcal{P}_{>0}^{<\omega} F_{\mho}(X)\right)$ and $\alpha \in$ $\operatorname{Con}_{f i}^{i d}\left(\mathcal{P}_{>0}^{<\omega} F_{\mho_{\tilde{\Theta}}}(X)\right)$ describes a subvariety $\mathcal{S}=\operatorname{HSP}\left(\left(\mathcal{P}_{>0}^{<\omega} \mathrm{F}_{\mho}(\mathrm{X}) / \Psi, \Omega, \cup\right)\right)$ of $\mathcal{S}_{\mho}$ such that $\widetilde{\Psi}=\widetilde{\Theta}$ and $\alpha=\delta_{\Psi}$. 
Consider the set

$$
\operatorname{Con}_{f i}^{i d}(\mho):=\bigcup_{\Theta \in \operatorname{Con}_{f i}^{9 i}\left(\mathcal{P}_{>0}^{<\infty} F_{\mho}(X)\right)} \operatorname{Con}_{f i}^{i d}\left(\mathcal{P}_{>0}^{<\omega} F_{\mho_{\tilde{\Theta}}}(X)\right) .
$$

One has

$$
\alpha \in \operatorname{Con}_{f i}^{i d}(\mho) \Leftrightarrow \exists \Theta \in \operatorname{Con}_{f i}^{\Re}\left(\mathcal{P}_{>0}^{<\omega} F_{\mho}(X)\right) \text { such that } \alpha \in \operatorname{Con}_{f i}^{i d}\left(\mathcal{P}_{>0}^{<\omega} F_{\mho_{\tilde{\Theta}}}(X)\right) .
$$

To stress the fact that the congruence $\alpha$ depends on $\Theta$ we denote it by $\alpha^{\widetilde{\Theta}}$. Now, define on the set $\operatorname{Con}_{f i}^{i d}(\mho)$ a binary relation $\preceq$ in the following way: for $\alpha^{\widetilde{\Theta}} \in$ $\operatorname{Con}_{f i}^{i d}\left(\mathcal{P}_{>0}^{<\omega} F_{\mho_{\Theta}}(X)\right)$ and $\beta^{\widetilde{\Psi}} \in \operatorname{Con}_{f i}^{i d}\left(\mathcal{P}_{>0}^{<\omega} F_{\mho_{\tilde{\Psi}}}(X)\right)$, with $\Theta, \Psi \in \operatorname{Con}_{f i}^{\Re i}\left(\mathcal{P}_{>0}^{<\omega} F_{\mho}(X)\right)$

$$
\begin{aligned}
& \alpha^{\widetilde{\Theta}} \preceq \beta^{\widetilde{\Psi}} \Leftrightarrow \widetilde{\Theta} \subseteq \widetilde{\Psi} \text { and } \forall\left(a_{1}, \ldots, a_{k}, b_{1}, \ldots, b_{m} \in F_{\mho}(X)\right) \\
& \left(\left\{a_{1} / \widetilde{\Theta}, \ldots, a_{k} / \widetilde{\Theta}\right\},\left\{b_{1} / \widetilde{\Theta}, \ldots, b_{m} / \widetilde{\Theta}\right\}\right) \in \alpha^{\widetilde{\Theta}} \Rightarrow \\
& \left(\left\{a_{1} / \widetilde{\Psi}, \ldots, a_{k} / \widetilde{\Psi}\right\},\left\{b_{1} / \widetilde{\Psi}, \ldots, b_{m} / \widetilde{\Psi}\right\}\right) \in \beta^{\widetilde{\Psi}} .
\end{aligned}
$$

Clearly, the relation $\preceq$ is a partial order.

Remark 5.10 Let $\Theta, \Lambda \in \operatorname{Con}_{f i}^{\Re i}\left(\mathcal{P}_{>0}^{<\omega} F_{\mho}(X)\right)$ and let $\Psi, \Gamma \in \operatorname{Con}_{f i}\left(\mathcal{P}_{>0}^{<\omega} F_{\mho}(X)\right)$ be such that $\widetilde{\Psi}=\widetilde{\Theta}$ and $\widetilde{\Gamma}=\widetilde{\Lambda}$. Then for the congruences $\delta_{\Psi} \in \operatorname{Con}_{f i}^{i d}\left(\mathcal{P}_{>0}^{<\omega} F_{\mho_{\tilde{\Theta}}}(X)\right)$, $\delta_{\Gamma} \in \operatorname{Con}_{f i}^{i d}\left(\mathcal{P}_{>0}^{<\omega} F_{\mho_{\tilde{\Lambda}}}(X)\right)$ one has

$$
\delta_{\Psi} \preceq \delta_{\Gamma} \quad \Leftrightarrow \quad \Psi \subseteq \Gamma .
$$

Theorem 5.11 The ordered set $\left(\operatorname{Con}_{f i}^{i d}(\mho), \preceq\right)$ is a complete lattice.

Let $I$ be a set and for each $i \in I, \alpha^{\widetilde{\Theta}_{i}} \in \operatorname{Con}_{f i}^{i d}\left(\mathcal{P}_{>0}^{<\omega} F_{\mho_{\widetilde{\Theta}_{i}}}(X)\right)$. The binary relations

$$
\begin{aligned}
& \delta_{\bigcap_{i \in I} \Delta_{a} \tilde{\Theta}_{i}} \subseteq \mathcal{P}_{>0}^{<\omega} F_{\mho}{ }_{i \in I} \widetilde{\Theta}_{i}(X) \times \mathcal{P}_{>0}^{<\omega} F_{\mho_{\bigcap \tilde{\Theta}_{i}}}(X) \text {, and } \\
& \delta_{i \in I} \Delta_{\alpha \tilde{\Theta}_{i}} \subseteq \mathcal{P}_{>0}^{<\omega} F_{\mho_{\tilde{\Upsilon}}}(X) \times \mathcal{P}_{>0}^{<\omega} F_{\mho_{\widetilde{\Upsilon}}}(X) \text {, where } \Upsilon:=\bigcap_{\Phi \in\left(\bigvee_{i \in I} \Delta_{\alpha^{\prime} \tilde{\Theta}_{i}}\right) / \Re} \Phi,
\end{aligned}
$$

are, respectively, the greatest lower bound and the least upper bound of $\left\{\alpha^{\widetilde{\Theta}_{i}}\right\}_{i \in I}$ with respect to $\preceq$.

Proof Let $\Theta, \Phi \in \operatorname{Con}_{f i}^{\Re i}\left(\mathcal{P}_{>0}^{<\omega} F_{\mho}(X)\right)$. By Lemma 5.6, for any $\alpha^{\widetilde{\Theta}} \in \operatorname{Con}_{f i}^{i d}(\mho)$ we have $\widetilde{\Delta}_{\alpha} \widetilde{\Theta}=\widetilde{\Theta}$. Then by Lemma 4.3 , one obtains

$$
\widehat{\bigcap_{i \in I} \Delta_{\alpha} \tilde{\Theta}_{i}}=\bigcap_{i \in I} \widetilde{\Delta}_{\alpha \tilde{\Theta}_{i}}=\bigcap_{i \in I} \widetilde{\Theta}_{i}=\widetilde{\bigcap_{i \in I} \Theta_{i}} \text { and } \widetilde{\bigvee_{i \in I} \Delta_{\alpha} \tilde{\Theta}_{i}}=\tilde{\Upsilon} \text {. }
$$

Hence, by Lemmas 5.5 and 5.6, the congruences $\delta_{\bigcap_{i \in I} \Delta_{\alpha} \tilde{\Theta}_{i}}$ and $\delta_{i \in I} \Delta_{\alpha} \tilde{\Theta}_{i}$ belong to the set $\operatorname{Con}_{f i}^{i d}(\mho)$. 
Obviously, for each $i \in I, \bigcap_{i \in I} \widetilde{\Theta}_{i} \subseteq \widetilde{\Theta}_{i}$. Moreover, for $t_{1}, \ldots, t_{k}, u_{1}, \ldots, u_{m} \in F_{\mho}(X)$

$$
\begin{aligned}
& \left(\left\{t_{1} / \bigcap_{i \in I} \widetilde{\Theta}_{i}, \ldots, t_{k} / \bigcap_{i \in I} \widetilde{\Theta}_{i}\right\},\left\{u_{1} / \bigcap_{i \in I} \widetilde{\Theta}_{i}, \ldots, u_{m} / \bigcap_{i \in I} \widetilde{\Theta}_{i}\right\}\right) \in \delta_{i \in I} \Delta_{\alpha} \widetilde{\Theta}_{i} \\
& \forall(i \in I) \quad\left(\left\{t_{1} / \widetilde{\Theta}_{i}, \ldots, t_{k} / \widetilde{\Theta}_{i}\right\},\left\{u_{1} / \widetilde{\Theta}_{i}, \ldots, u_{m} / \widetilde{\Theta}_{i}\right\}\right) \in \alpha^{\widetilde{\Theta}_{i}} .
\end{aligned}
$$

This implies that for each $i \in I$,

$$
\delta_{i \in I} \Delta_{\alpha} \widetilde{\Theta}_{i} \preceq \alpha^{\widetilde{\Theta}_{i}}
$$

Now let $\gamma^{\widetilde{\Phi}} \in \operatorname{Con}_{f i}^{i d}\left(\mathcal{P}_{>0}^{<\omega} F_{\mho_{\widetilde{\Phi}}}(X)\right)$ and for each $i \in I, \gamma \gamma^{\widetilde{\Phi}} \preceq \alpha^{\widetilde{\Theta}_{i}}$. Then, for each $i \in I, \widetilde{\Phi} \subseteq \widetilde{\Theta}_{i}$, and consequently, $\widetilde{\Phi} \subseteq \bigcap_{i \in I} \widetilde{\Theta}_{i}$. Further, for any $t_{1}, \ldots, t_{k}, u_{1}, \ldots, u_{m} \in$ $F_{\mho}(X)$

$$
\begin{aligned}
& \left(\left\{t_{1} / \widetilde{\Phi}, \ldots, t_{k} / \widetilde{\Phi}\right\},\left\{u_{1} / \widetilde{\Phi}, \ldots, u_{m} / \widetilde{\Phi}\right\}\right) \in \gamma^{\widetilde{\Phi}} \Rightarrow \\
& \forall(i \in I) \quad\left(\left\{t_{1} / \widetilde{\Theta}_{i}, \ldots, t_{k} / \widetilde{\Theta}_{i}\right\},\left\{u_{1} / \widetilde{\Theta}_{i}, \ldots, u_{m} / \widetilde{\Theta}_{i}\right\}\right) \in \alpha^{\widetilde{\Theta}_{i}} \Rightarrow \\
& \forall(i \in I)\left(\left\{t_{1}, \ldots, t_{k}\right\},\left\{u_{1}, \ldots, u_{m}\right\}\right) \in \Delta_{\alpha} \widetilde{\Theta}_{i} \Rightarrow \\
& \left(\left\{t_{1}, \ldots, t_{k}\right\},\left\{u_{1}, \ldots, u_{m}\right\}\right) \in \bigcap_{i \in I} \Delta_{\alpha}{\widetilde{\Theta_{i}}}_{i} \Leftrightarrow \\
& \left(\left\{t_{1} / \bigcap_{i \in I} \widetilde{\Theta}_{i}, \ldots, t_{k} / \bigcap_{i \in I} \widetilde{\Theta}_{i}\right\},\left\{u_{1} / \bigcap_{i \in I} \widetilde{\Theta}_{i}, \ldots, u_{m} / \bigcap_{i \in I} \widetilde{\Theta}_{i}\right\}\right) \in \delta_{\bigcap_{i \in I} \Delta_{\alpha} \widetilde{\Theta}_{i}} .
\end{aligned}
$$

This shows the relation $\delta_{i \in I} \Delta_{\alpha} \widetilde{\Theta}_{i}$ is the greatest lower bound of $\left\{\alpha^{\widetilde{\Theta}_{i}}\right\}_{i \in I}$ with respect to $\preceq$.

Now note that for each $i \in I$,

$$
\widetilde{\Delta}_{\alpha \tilde{\Theta}_{i}}=\widetilde{\Theta}_{i} \subseteq \bigvee_{i \in I} \widetilde{\Theta}_{i}=\bigvee_{i \in I} \widetilde{\Delta}_{\alpha \tilde{\Theta}_{i}} \subseteq \widetilde{\bigvee_{i \in I} \Delta_{\alpha} \tilde{\Theta}_{i}}=\widetilde{\Upsilon}
$$

and for $t_{1}, \ldots, t_{k}, u_{1}, \ldots, u_{m} \in F_{\mho}(X)$ we have

$$
\begin{aligned}
& \left(\left\{t_{1} / \widetilde{\Theta}_{i}, \ldots, t_{k} / \widetilde{\Theta}_{i}\right\},\left\{u_{1} / \widetilde{\Theta}_{i}, \ldots, u_{m} / \widetilde{\Theta}_{i}\right\}\right) \in \alpha^{\widetilde{\Theta}_{i}} \Leftrightarrow \\
& \left(\left\{t_{1}, \ldots, t_{k}\right\},\left\{u_{1}, \ldots, u_{m}\right\}\right) \in \Delta_{\alpha \widetilde{\Theta}_{i}} \Rightarrow \\
& \left(\left\{t_{1}, \ldots, t_{k}\right\},\left\{u_{1}, \ldots, u_{m}\right\}\right) \in \bigvee_{i \in I} \Delta_{\alpha} \widetilde{\Theta}_{i} \Rightarrow \\
& \left(\left\{t_{1} / \widetilde{\Upsilon}, \ldots, t_{k} / \widetilde{\Upsilon}\right\},\left\{u_{1} / \widetilde{\Upsilon}, \ldots, u_{m} / \widetilde{\Upsilon}\right\}\right) \in \delta_{i \in I} \Delta_{\alpha} \widetilde{\Theta}_{i} \cdot
\end{aligned}
$$

Thus, for each $i \in I, \alpha^{\widetilde{\Theta}_{i}} \preceq \delta_{i \in I} \Delta_{\alpha} \widetilde{\Theta}_{i}$. 
Finally, let $\gamma^{\widetilde{\Phi}} \in \operatorname{Con}_{f i}^{i d}\left(\mathcal{P}_{>0}^{<\omega} F_{\mho_{\widetilde{\Phi}}}(X)\right)$ and for each $i \in I, \alpha^{\widetilde{\Theta}_{i}} \preceq \gamma^{\widetilde{\Phi}}$. Then, for each $i \in I, \widetilde{\Theta}_{i} \subseteq \widetilde{\Phi}$ and for $t_{1}, \ldots, t_{k}, u_{1}, \ldots, u_{m} \in F_{\mho}(X)$

$$
\begin{gathered}
\left(\left\{t_{1}, \ldots, t_{k}\right\},\left\{u_{1}, \ldots, u_{m}\right\}\right) \in \Delta_{\alpha} \widetilde{\Theta}_{i} \Leftrightarrow \\
\left(\left\{t_{1} / \widetilde{\Theta}_{i}, \ldots, t_{k} / \widetilde{\Theta}_{i}\right\},\left\{u_{1} / \widetilde{\Theta}_{i}, \ldots, u_{m} / \widetilde{\Theta}_{i}\right\}\right) \in \alpha^{\widetilde{\Theta}_{i}} \Rightarrow \\
\left(\left\{t_{1} / \widetilde{\Phi}, \ldots, t_{k} / \widetilde{\Phi}\right\},\left\{u_{1} / \widetilde{\Phi}, \ldots, u_{m} / \widetilde{\Phi}\right\}\right) \in \gamma^{\widetilde{\Phi}} \Leftrightarrow \\
\left(\left\{t_{1}, \ldots, t_{k}\right\},\left\{u_{1}, \ldots, u_{m}\right\}\right) \in \Delta_{\gamma} \widetilde{\Phi} .
\end{gathered}
$$

Then, for each $i \in I, \Delta_{\alpha} \widetilde{\Theta}_{i} \subseteq \Delta_{\gamma} \tilde{\Phi}$. Hence $\bigvee_{i \in I} \Delta_{\alpha^{\tilde{\Theta}_{i}}} \subseteq \Delta_{\gamma} \tilde{\Phi}$, which implies

$$
\widetilde{\Upsilon}=\bigvee_{i \in I} \Delta_{\alpha} \tilde{\Theta}_{i} \subseteq \widetilde{\Delta}_{\gamma \widetilde{\Phi}}=\widetilde{\Phi} .
$$

Moreover, by Lemma 5.7 we have

$$
\begin{aligned}
& \left(\left\{t_{1} / \widetilde{\Upsilon}, \ldots, t_{k} / \widetilde{\Upsilon}\right\},\left\{u_{1} / \widetilde{\Upsilon}, \ldots, u_{m} / \widetilde{\Upsilon}\right\}\right) \in \delta_{i \in I} \Delta_{\alpha^{\Theta_{i}}} \Leftrightarrow \\
& \left(\left\{t_{1}, \ldots, t_{k}\right\},\left\{u_{1}, \ldots, u_{m}\right\}\right) \in \bigvee_{i \in I} \Delta_{\alpha} \tilde{\Theta}_{i} \Rightarrow \\
& \left(\left\{t_{1}, \ldots, t_{k}\right\},\left\{u_{1}, \ldots, u_{m}\right\}\right) \in \Delta_{\gamma} \tilde{\Phi} \Leftrightarrow \\
& \left(\left\{t_{1} / \widetilde{\Phi}, \ldots, t_{k} / \widetilde{\Phi}\right\},\left\{u_{1} / \widetilde{\Phi}, \ldots, u_{m} / \widetilde{\Phi}\right\}\right) \in \delta_{\Delta_{\gamma} \widetilde{\Phi}}=\gamma^{\widetilde{\Phi}},
\end{aligned}
$$

which means the relation $\delta_{i \in I} \Delta_{\alpha} \widetilde{\Theta}_{i}$ is the least upper bound of $\left\{\alpha^{\widetilde{\Theta}_{i}}\right\}_{i \in I}$, and completes the proof.

By Theorem 5.11 we obtain a full description of the lattice of all subvarieties of the variety $\mathcal{S}_{\mho}$.

Let $\mathcal{L}\left(\mathcal{S}_{\mho}\right)$ denote the set of all subvarieties of the variety $\mathcal{S}_{\mho}$. As we have already noticed each subvariety $\mathcal{S}$ of $\mathcal{S}_{\mho}$ may be uniquely described by two congruences: $\Theta \in \operatorname{Con}_{f i}^{\Re}\left(\mathcal{P}_{>0}^{<\omega} F_{\mho}(X)\right)$ and $\alpha^{\widetilde{\Theta}} \in \operatorname{Con}_{f i}^{i d}\left(\mathcal{P}_{>0}^{<\omega} F_{\mho_{\Theta}}(X)\right)$. Hence, we can denote each subvariety in the set $\mathcal{L}\left(\mathcal{S}_{\mho}\right)$ by $\mathcal{S}_{\mho_{\tilde{\Theta}}}^{\alpha_{\tilde{\Theta}}}$. Thus, one has

$$
\mathcal{L}\left(\mathcal{S}_{\mho}\right)=\left\{\mathcal{S}_{\mho_{\tilde{\Theta}}}^{\alpha^{\widetilde{\Theta}}} \mid \Theta \in \operatorname{Con}_{f i}^{\Re}\left(\mathcal{P}_{>0}^{<\omega} F_{\mho}(X)\right), \alpha^{\widetilde{\Theta}} \in \operatorname{Con}_{f i}^{i d}\left(\mathcal{P}_{>0}^{<\omega} F_{\mho_{\tilde{\Theta}}}(X)\right)\right\} .
$$

Theorem 5.12 The lattice $\left(\mathcal{L}\left(\mathcal{S}_{\mho}\right), \subseteq\right)$ of all subvarieties of the variety $\mathcal{S}_{\mho}$ is dually isomorphic to the lattice $\left(\operatorname{Con}_{f i}^{i d}(\mho), \preceq\right)$.

For any $\mathcal{S}_{\mho_{\tilde{\Theta}}}^{\alpha_{\tilde{\Theta}}^{\tilde{E}}}, \mathcal{S}_{\mho_{\tilde{\Psi}}}^{\beta_{\tilde{\Psi}}^{\tilde{T}}} \in \mathcal{L}\left(\mathcal{S}_{\mho}\right)$ we have:

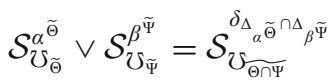

and

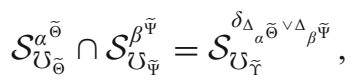


Fig. 2 The lattice $\left(\mathcal{L}\left(\mathcal{S}_{\mho}\right), \subseteq\right)$

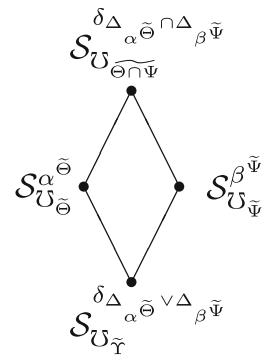

where $\Upsilon:=\bigcap_{\Phi \in\left(\Delta_{\alpha} \widetilde{\Theta} \vee \Delta_{\beta} \widetilde{\Psi}\right) / \Re} \Phi$, (see Fig. 2).

Theorem 5.12 allows to describe the subvariety lattice $\mathcal{L}\left(\mathcal{S}_{\mho}\right)$ without knowledge of the set $\operatorname{Con}_{f i}\left(\mathcal{P}_{>0}^{<\omega} F_{\mho}(X)\right)$. But, if we know the latter, each congruence $\alpha^{\widetilde{\Theta}} \in \operatorname{Con}_{f i}^{i d}\left(\mathcal{P}_{>0}^{<\omega} F_{\mho_{\tilde{\Theta}}}(X)\right)$ may be replaced by the congruence $\delta_{\Psi}$, for $\Psi \in$ $\operatorname{Con}_{f i}\left(\mathcal{P}_{>0}^{<\omega} F_{\mho}(X)\right)$ such that $\widetilde{\Psi}=\widetilde{\Theta}$. Then, Theorem 5.12 may be considerably simplified.

Corollary 5.13 For any $\mathcal{S}_{\mho_{\tilde{\Theta}}}^{\delta_{\Psi}}, \mathcal{S}_{\mho_{\tilde{\Gamma}}}^{\delta_{\Phi}} \in \mathcal{L}\left(\mathcal{S}_{\mho}\right)$ we have:

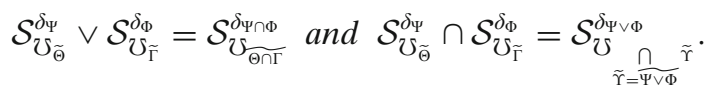

\section{The Lattice $\mathcal{L}\left(\mathcal{S}_{\mho}\right)$-Practical Computations}

Now, for a subvariety $\mathcal{V} \subseteq \mathcal{V}$, we will present how to find the lattice $\left(\mathcal{L}\left(\mathcal{S}_{\mathcal{V}}\right), \subseteq\right)$ of all subvarieties of the variety $\mathcal{S}_{\mathcal{V}}$, knowing the lattice $(\mathcal{L}(\mathcal{V}), \subseteq)$. Of course, any lattice $\left(\mathcal{L}\left(\mathcal{S}_{\mathcal{V}}\right), \subseteq\right)$ is a sublattice of the lattice $\left(\mathcal{L}\left(\mathcal{S}_{\mho}\right), \subseteq\right)$.

On the other hand, Example 3.5 shows that not for each subvariety $\mathcal{V} \subseteq \mathcal{V}$, the variety $\mathcal{S}_{\mathcal{V}}$ is uniquely defined. Let us consider two sets

$$
\begin{aligned}
& \operatorname{Con}_{\mathcal{V}}:=\left\{\psi \in \operatorname{Con}_{f i}\left(\mathcal{P}_{>0}^{<\omega} F_{\mathcal{V}}(X)\right) \mid \tilde{\psi}=i d_{F_{\mathcal{V}}(X)} \text { and }\left(\mathcal{P}_{>0}^{<\omega} F_{\mathcal{V}}(X) / \psi, \Omega\right) \in \mathcal{V}\right\} \text { and } \\
& \mathfrak{O}_{\mathcal{V}}:=\left\{\mathcal{W} \subseteq \mathcal{V} \mid \operatorname{Con}_{\mathcal{W}} \neq \emptyset\right\} .
\end{aligned}
$$

By Lemma 5.6, if $\operatorname{Con}_{\mathcal{V}} \neq \emptyset$ then $\mathcal{S}_{\mathcal{V}}$ is not equal to $\mathcal{S}_{\mathcal{W}}$ for any proper subvariety $\mathcal{W} \subset \mathcal{V}$. Therefore, to find the lattice $\left(\mathcal{L}\left(\mathcal{S}_{\mathcal{V}}\right), \subseteq\right)$, Theorems 5.9, 5.11 and 5.12 lead us to the procedure described on Fig. 3.

By Theorems 5.11 and 5.12, the algebra $\left(\bigcup_{\mathcal{W} \in \mathcal{O}_{\mathcal{V}}} \operatorname{Con}_{\mathcal{W}}, \sqcap, \sqcup\right)$, with

$$
\alpha^{\mathcal{W}_{1}} \sqcap \beta^{\mathcal{W}_{2}}:=\delta_{\Delta_{\alpha} \mathcal{W}_{1} \cap \Delta_{\beta} \mathcal{W}_{2}} \in \operatorname{Con}_{\mathcal{W}_{1} \vee \mathcal{W}_{2}}
$$

and

$$
\alpha^{\mathcal{W}_{1}} \sqcup \beta^{\mathcal{W}_{2}}:=\delta_{\Delta_{\alpha} \mathcal{W}_{1} \vee \Delta_{\beta} \mathcal{W}_{2}} \in \operatorname{Con}_{\mathcal{U}}
$$




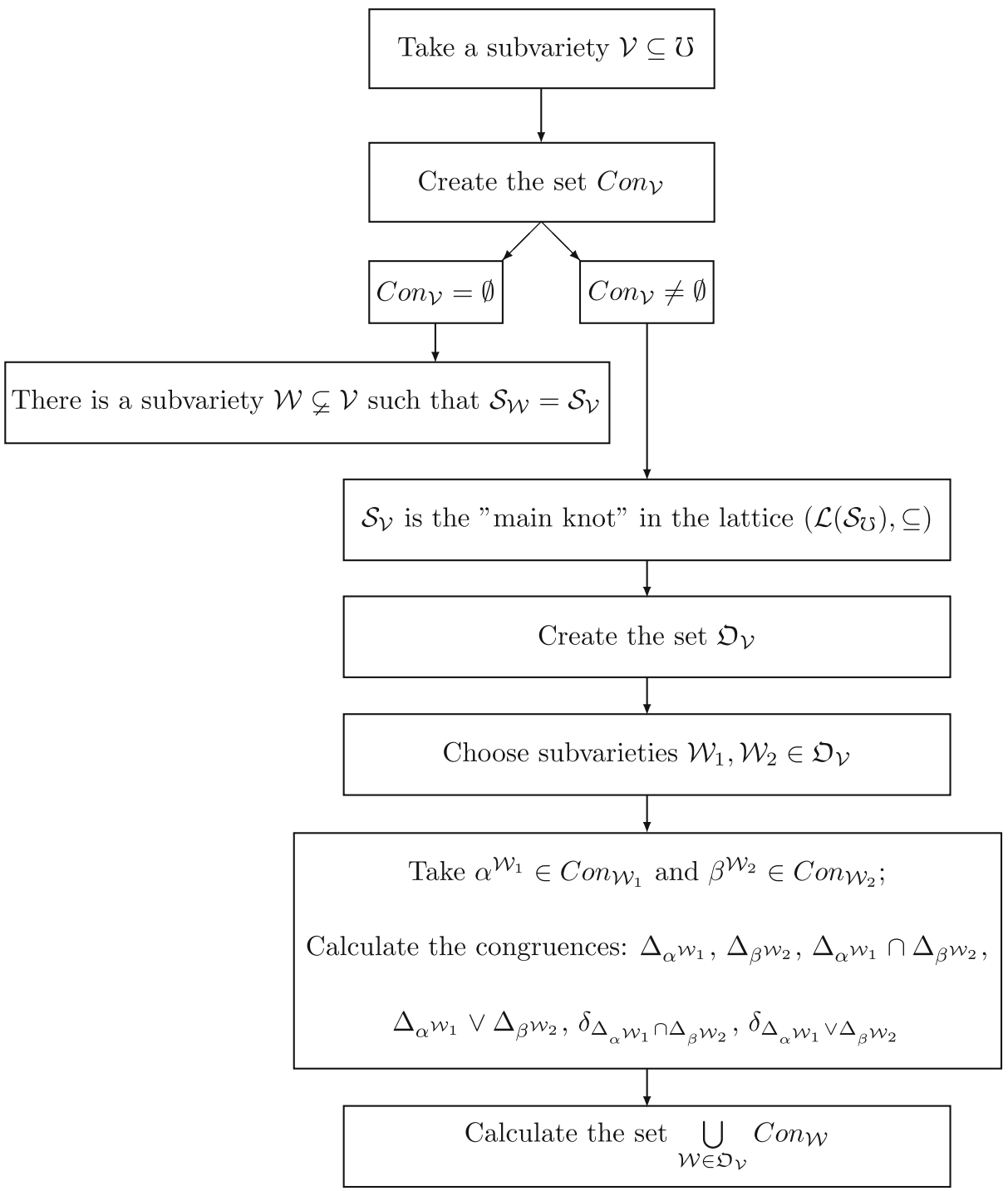

Fig. 3 Algorithm of finding the lattice $\mathcal{L}\left(\mathcal{S}_{\mathcal{V}}\right)$

where $\mathcal{U}=\operatorname{HSP}\left(\left(\mathcal{P}_{>0}^{<\omega}\left(F_{\mathcal{V}}(X) / \Delta_{\alpha}{\widetilde{\mathcal{W}_{1}} \vee \Delta_{\beta}}_{\mathcal{W}_{2}}\right), \Omega, \cup\right)\right)$, is a lattice dually isomorphic to the complete lattice $\left(\mathcal{L}\left(\mathcal{S}_{\mathcal{V}}\right), \subseteq\right)$ of all subvarieties of the variety $\mathcal{S}_{\mathcal{V}}$.

Example 6.1 Let $\mho$ be the variety of all binary algebras $(A, \cdot)$ and $\mathcal{L} \mathcal{Z} \subseteq \mho$ be the subvariety of left-zero semigroups. It is known that $\mathcal{L} \mathcal{Z}$-free algebra $\left(F_{\mathcal{L Z}}(X), \cdot\right)$ on a set $X$ is isomorphic to the left-zero groupoid $(X, \cdot)$. It is also easy to notice $\left(\mathcal{P}_{>0}^{<\omega} X, \cdot\right) \in \mathcal{L} \mathcal{Z}$

By Corollary 3.3, for any $\mathcal{V} \subseteq \mathcal{V}$, the semilattice ordered algebra $\left(\mathcal{P}_{>0}^{<\omega} F_{\mathcal{V}}(X), \cdot, \cup\right)$ is free over a set $X$ in the variety $\mathcal{S}_{\mathcal{V}}$ if and only if $\left(\mathcal{P}_{>0}^{<\omega} F_{\mathcal{V}}(X), \cdot, \cup\right) \in \mathcal{S}_{\mathcal{V}}$. Then the 
algebra $\left(\mathcal{P}_{>0}^{<\omega} X, \cdot, \cup\right)$ is free in the variety $\mathcal{S}_{\mathcal{L Z}}$ of all semilattice ordered $\mathcal{L} \mathcal{Z}$-algebras. Moreover, the algebra $\left(\mathcal{P}_{>0}^{<\omega} X, \cdot, \cup\right)$ is generated by the set $\{\{x\} \mid x \in X\}$.

Let $Q=\left\{q_{1}, \ldots, q_{k}\right\} \subseteq X$ and $\alpha$ be an endomorphism of $\left(\mathcal{P}_{>0}^{<\omega} X, \cdot, \cup\right)$. Because each homomorphism is uniquely defined on generators of an algebra, $\alpha(Q)=$ $\alpha\left(\left\{q_{1}\right\}\right) \cup \ldots \cup \alpha\left(\left\{q_{k}\right\}\right)$ might be any finite subset of $X$. This implies $\operatorname{Con}_{\mathcal{L Z}}=$ $\left\{i d_{\mathcal{P}_{>0}^{<\omega} X}\right\}$ and shows there are only two subvarieties of the variety $\mathcal{S}_{\mathcal{L Z}}: \mathcal{S}_{\mathcal{L Z}}$ itself and trivial one.

Example 6.2 Let $\mho \mathcal{S}$ be the variety of all binary algebras $(A, \cdot), \mathcal{S G}$ be the variety of all semigroups and $\mathcal{S} \mathcal{L}$ denote the variety of all semilattices.

By results of Grätzer and Lakser ([6, Proposition 1]), the algebra $\left(\mathcal{P}_{>0}^{<\omega} F_{\mathcal{S L}}(X), \cdot\right)$ is a commutative semigroup, but it is not idempotent. Hence, the algebra $\left(\mathcal{P}_{>0}^{<\omega} F_{\mathcal{S} \mathcal{L}}(X), \cdot\right)$ does not belong to the variety $\mathcal{S}_{\mathcal{S} \mathcal{L}}$, and consequently by Corollary 3.3, it is not free in $\mathcal{S}_{\mathcal{S} \mathcal{L}}$.

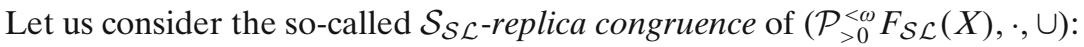

$$
\Phi_{\mathcal{S}_{\mathcal{S}}}(X):=\bigcap\left\{\phi \in \operatorname{Con}\left(\mathcal{P}_{>0}^{<\omega} F_{\mathcal{S L}}(X), \cdot, \cup\right) \mid\left(\mathcal{P}_{>0}^{<\omega} F_{\mathcal{S L}}(X) / \phi, \cdot, \cup\right) \in \mathcal{S}_{\mathcal{S L}}\right\}
$$

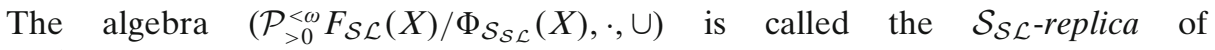
$\left(\mathcal{P}_{>0}^{<\omega} F_{\mathcal{S L}}(X), \cdot, \cup\right)$.

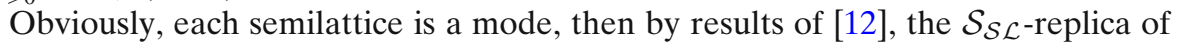
the algebra $\left(\mathcal{P}_{>0}^{<\omega} F_{\mathcal{S L}}(X), \cdot, \cup\right)$ is free over a set $X$ in the variety $\mathcal{S}_{\mathcal{S L}}$.

The free semilattice $\left(F_{\mathcal{S}}(X), \cdot\right)$ over a set $X$ is isomorphic to the algebra

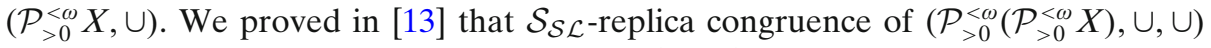
is defined in the following way: for $Q, R \in \mathcal{P}_{>0}^{<\omega}\left(\mathcal{P}_{>0}^{<\omega} X\right)$

$$
Q \Phi_{\mathcal{S}_{\mathcal{S}}}(X) R \Leftrightarrow\langle Q\rangle=\langle R\rangle,
$$

where $\langle S\rangle$ denotes the subalgebra of $\left(\mathcal{P}_{>0}^{<\omega} X, \cup\right)$ generated by $S$.

This shows the free algebra over a set $X$ in the variety $\mathcal{S}_{\mathcal{S} \mathcal{L}}$ is isomorphic to the algebra $\left(S\left(\mathcal{P}_{>0}^{<\omega} X\right),+\right)$ of all non empty, finitely generated subalgebras of the algebra $\left(\mathcal{P}_{>0}^{<\omega} X, \cup\right)$, with $S_{1}+S_{2}:=\left\langle S_{1} \cup S_{2}\right\rangle$.

By results of Kuřil and Polák ([9]), there are three more non-trivial fully invariant congruences of $\left(\mathcal{P}_{>0}^{<\omega}\left(\mathcal{P}_{>0}^{<\omega} X\right), \cup, \cup\right)$ greater than $\Phi_{\mathcal{S}_{\mathcal{S L}}}(X)$, which belong to the set

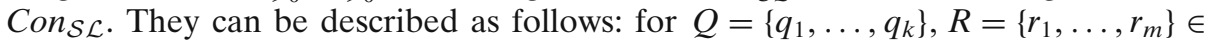
$\mathcal{P}_{>0}^{<\omega}\left(\mathcal{P}_{>0}^{<\infty} X\right)$

$$
\begin{aligned}
& Q \Phi_{1} R \Leftrightarrow \\
& \forall(q \in Q) \exists(r \in R) r \subseteq q \subseteq r_{1} \cup \ldots \cup r_{m} \text { and } \forall(r \in R) \exists(q \in Q) \quad q \subseteq r \subseteq q_{1} \cup \ldots \cup q_{k}, \\
& Q \Phi_{2} R \Leftrightarrow \\
& \forall(q \in Q) q \subseteq r_{1} \cup \ldots \cup r_{m} \text { and } \forall(r \in R) r \subseteq q_{1} \cup \ldots \cup q_{k}, \\
& Q \Phi_{3} R \Leftrightarrow \\
& \forall(q \in Q) \exists(r \in R) \quad r \subseteq q \text { and } \forall(r \in R) \exists(q \in Q) \quad q \subseteq r .
\end{aligned}
$$

This confirmed previous results of McKenzie and Romanowska (see [10]) that there are exactly five subvarieties of the variety $\mathcal{S}_{\mathcal{S L}}$. 
Acknowledgement We wish to thank the referee for an unusually careful reading of the paper and pointing out several weak places.

Open Access This article is distributed under the terms of the Creative Commons Attribution License which permits any use, distribution, and reproduction in any medium, provided the original author(s) and the source are credited.

\section{References}

1. Bloom, S.L.: Varieties of ordered algebras. J. Comput. Syst. Sci. 13, 200-212 (1976)

2. Brink, C.: Power structures. Algebra Univers. 30(2), 177-216 (1993)

3. Czédli, G., Lenkehegyi, A.: On classes of ordered algebras and quasiorder distributivity. Acta Sci. Math. 46, 41-54 (1983)

4. Fuchs, L.: On partially ordered algebras I. Colloq. Math. 14, 113-130 (1966)

5. Ghosh, S., Pastijn, F., Zhao, X.Z.: Varieties generated by ordered bands I. Order 22, 109-128 (2005)

6. Grätzer, G., Lakser, H.: Identities for globals (complex algebras) of algebras. Colloq. Math. 56, 19-29 (1988)

7. Jónsson, B., Tarski, A.: Boolean algebras with operators I. Am. J. Math. 73, 891-939 (1951)

8. Kearnes, K.: Semilattice modes I: the associated semiring. Algebra Univers. 34, 220-272 (1995)

9. Kuřil, M., Polák, L.: On varieties of semilattice-ordered semigroups. Semigroup Forum 71, 27-48 (2005)

10. McKenzie, R., Romanowska, A.: Varieties of - - distributive bisemilattices. Contrib. Gen. Algebra (Proc. Klagenfurt Conf. 1978) 1, 213-218 (1979)

11. Pastijn, F., Zhao, X.Z.: Varieties of idempotent semirings with commutative addition. Algebra Univers. 54, 301-321 (2005)

12. Pilitowska, A., Zamojska-Dzienio, A.: Representation of modals. Demonstr. Math. 44(3), 535556 (2011)

13. Pilitowska, A., Zamojska-Dzienio, A.: On some congruences of power algebras. Cent. Eur. J. Math. 10(3), 987-1003 (2012)

14. Romanowska, A.B., Roszkowska, B.: On some groupoid modes. Demonstr. Math. 20(1-2), 277290 (1987)

15. Romanowska, A.B., Smith, J.D.H.: Modal Theory. Heldermann Verlag, Berlin (1985)

16. Romanowska, A.B., Smith, J.D.H.: Modes. World Scientific, Singapore (2002)

17. Ślusarska, K.: Distributive differential modals. Discuss. Math. Gen. Algebra Appl. 28, 29-47 (2008)

18. Zhao, X.Z.: Idempotent semirings with a commutative additive reducts. Semigroup Forum 64, 289-296 (2002) 